\title{
Le système semencier céréalier au Burkina Faso : dépendance de sentier et trajectoires d'évolution de 1970 à 2020
}

\section{Anny Lucrèce Nlend Nkott et Ludovic Temple}

\section{(2) OpenEdition}

Journals

Édition électronique

URL : https://journals.openedition.org/ei/6798

DOI : 10.4000/ei.6798

ISSN : 2553-1891

Éditeur

Association Économie et Institutions

Référence électronique

Anny Lucrèce Nlend Nkott et Ludovic Temple, «Le système semencier céréalier au Burkina Faso : dépendance de sentier et trajectoires d'évolution de 1970 à 2020 », Économie et institutions [En ligne], 29 | 2021, mis en ligne le 01 décembre 2021, consulté le 02 février 2022. URL : http:// journals.openedition.org/ei/6798 ; DOI : https://doi.org/10.4000/ei.6798

Ce document a été généré automatiquement le 2 février 2022.

Revue Économie et institutions 


\title{
Le système semencier céréalier au Burkina Faso : dépendance de sentier et trajectoires d'évolution de 1970 à 2020
}

\author{
Anny Lucrèce Nlend Nkott et Ludovic Temple
}

\section{NOTE DE L'AUTEUR}

Financement : cette étude a été réalisée dans le cadre du projet COEX financé par la fondation Agropolis, et qui porte sur la gouvernance des systèmes semenciers en Afrique de l'Ouest

Remerciements: nous remercions tous les burkinabé qui ont répondu à nos questions. Nous remercions aussi Eveline Compaoré, sociologue à l'Institut de l'Environnement et de Recherche agricoles (INERA), pour son aide dans la mise en relation avec les acteurs.

\section{Introduction}

1 Depuis les années 1940 et la révolution verte portée par les CGIAR (Consultative Group on International Agricultural Research) dans les pays en développement (Temple, Chiffoleau et Touzard, 2018), l'innovation variétale est gouvernée par des acteurs de la recherche publique et privée, des services publics nationaux (Manzelli et Laghetti, 2014) et des firmes agrochimiques internationales (Bayer-Monsanto, Dupont Pioneer, Syngenta). Ce système d'acteurs est qualifié par la littérature de «système semencier formel » ou « officiel» (Almekinders, 2000 ; Almekinders, Louwaars et De Bruijn, 1994 ; Christinck et al., 2014; Louwaars et al., 2011). Il est structuré par des normes au niveau des critères (distinction, homogénéité et stabilité) requis pour la création de nouvelles variétés, qui ont fait le succès de la spécialisation de l'agriculture des pays industriels. Cette 
gouvernance est traversée par deux controverses sociotechniques qui s'intensifient. Premièrement, on observe un développement accru des innovations biotechnologiques, qui sont promues comme la réponse aux enjeux de développement durable (Moedas, 2017). Deuxièmement, une contestation sociétale du système dit formel voit le jour au début des années 2000. Cette contestation est portée par la société civile, à travers des mouvements de préservation des semences dites paysannes, locales ou traditionnelles (Demeulenaere, 2013). Les concepts de "biodiversité " et "souveraineté alimentaire » sont ainsi mobilisés pour montrer les relations entre les capacités d'innovation des sociétés agraires et la diversité des modes de production agricole à l'échelle mondiale. Plus qu'un enjeu environnemental, la «sécurité semencière " devient un problème politique et un sujet de discussion aux niveaux local, national et intergouvernemental (Groupe Crucible II et Foundation, 2001)

2 Sur le plan juridique, l'accaparement du droit du vivant par le mécanisme de la propriété intellectuelle obéit à des logiques commerciales des firmes, et génère une dépendance des producteurs des pays en développement (PED) à l'égard des innovations biotechnologiques issues des pays industrialisés (Laperche, 2009). Cet accaparement mettrait en cause les droits des agriculteurs à utiliser, échanger librement les semences, et conduit au besoin de règlementer l'accès aux ressources génétiques en application du Traité International sur les ressources Phytogénétiques pour l'Agriculture et l'Alimentation (TIRPAA). L'une des interrogations porte ainsi sur la capacité de la propriété intellectuelle à préserver la biodiversité (Groupe Crucible II et Foundation, 2001). Sur le plan géopolitique, le renforcement des Droits de Propriété Intellectuelle (DPI) présente le risque d'accroître le coût d'acquisition de la technologie pour les centres nationaux de recherche des PED. Cela renforcerait l'écart de connaissances entre pays industrialisés et en développement, et aboutirait à ce que Ismail Serageldin, ex vice-président de la banque mondiale qualifia «d'apartheid scientifique » (Serageldin, 1999, 2011).

3 Ce contexte pousse à s'interroger sur les conditions d'émergence d'institutions qui régulent l'accès au vivant, et structurent les perceptions et valeurs collectives citoyennes, centraux dans la gouvernance des trajectoires technologiques. Ces conditions sont particulièrement questionnées dans les PED où, les niveaux de pauvreté et d'insécurité alimentaire sont utilisés pour justifier le soutien à la globalisation d'un modèle agricole fondé sur celui des pays industriels. L'étude s'intéresse au cas du Burkina Faso, un pays d'Afrique de l'ouest pour lequel l'agriculture représente $40 \% \mathrm{du}$ PIB, et rassemble près de $86 \%$ de la population active (Ministère de l'agriculture et des aménagements hydrauliques, 2010).

4 Dans une lecture veblenienne, nous montrons qu'il existe une dépendance de sentier dans le système des semences certifiées, avec une interdépendance des acteurs et des cadres institutionnels aux niveaux national, sous-régional et international. Ensuite, nous analysons comment cette dépendance ne génère pas de verrouillage institutionnel. Tout d'abord, nous questionnons la pertinence de la dichotomie entre secteurs dits formel et informel, qui représente les systèmes des semences certifiées et des semences paysannes. 


\section{Un cadre conceptuel structuré par l'économie néo- institutionnelle et évolutionniste}

\subsection{La controverse analytique formel et informel dans le secteur semencier}

La notion «d'informel» émerge dans les années 70, dans les travaux du Bureau International du Travail (BIT) au Kenya (BIT, 1972, cité par Hugon, 2014). Après le premier choc pétrolier (1973-1974), de nombreux salariés perdirent leur emploi, ce qui donna lieu à un accroissement du taux de chômage (10\% de la population active) dans les pays industriels (Pesqueux, 2012). Cela a eu pour corollaire le développement de l'emploi indépendant et l'auto-emploi. Cependant, ce chômage n'était que faiblement observé dans les PED. Le BIT au Kenya avait donc pour mission d'expliquer comment et pourquoi l'absence de création d'emplois dans le secteur moderne n'avait pas provoqué une augmentation insoutenable du chômage dans PED (BIT, 1972, cité par Hugon, 2014). Le concept «d'informel» qui émerge à l'époque s'appliquait ainsi de manière spécifique aux PED. La Conférence Internationale des Statisticiens du Travail (CIST) en 1993 va définir le secteur informel comme « un ensemble d'unités produisant des biens et services, en vue principalement de créer des emplois et des revenus pour les personnes concernées. Ces unités, ayant un faible niveau d'organisation, opèrent à petite échelle, avec peu ou pas de division entre le travail et le capital. Les relations de travail sont fondées sur l'emploi occasionnel, les relations de parenté ou les relations personnelles, plutôt que sur des contrats comportant des garanties en bonne et due forme » (BIT, 1993, cité par Pesqueux, 2012).

6 Appliqués aujourd'hui à la caractérisation du secteur semencier, les adjectifs « formel » et «informel » tendent à différencier les organisations ou systèmes d'acteurs qui seraient performants du point de vue de l'augmentation de la production, de ceux qui ne le sont pas. Cette différentiation est controversée pour diverses raisons. Premièrement, dans les PED le secteur informel représente 50 à 70\% de la population (Pesqueux, 2012), ce qui veut dire que le secteur formel est l'exception, mais qui donne droit à la normalisation. Dans l'agriculture et la production semencière spécifiquement, le secteur informel reste la source majeure d'approvisionnement en semences à travers la sélection massale, et le troc (Gill et al., 2013 ; McGuire et Sperling, 2016 ; Sperling et McGuire, 2010). Deuxièmement, la notion «d'informel » institue un dualisme entre le secteur capitaliste qui serait "moderne" et source de progrès, et le secteur traditionnel ou de subsistance qui serait «archaïque " (Lewis, 1954, cité par Hugon, 2014). Ce dualisme considère l'informel comme un accident, au regard de la force institutionnelle du marché ; une sorte de défaillance de l'Etat qui aurait vocation à ne pas perdurer, et à être récupérée par l'économie marchande. Or, dans un contexte mondial où la biodiversité permet l'adaptation des sociétés humaines aux enjeux environnementaux et sanitaires, l'homogénéisation des mécanismes de coordination est de moins en moins acceptable. Nous affirmons que la pluralité des normes, des règles, donne lieu à une pluralité de modes de coordination de systèmes complexes. En outre, La vision dualiste en juxtaposant des catégories ne met pas en évidence l'imbrication ou l'hybridation de catégories (Hugon, 2014), ce dont nous rendons compte dans nos résultats. 
7 Enfin, si le secteur informel est souvent décrit comme souterrain, illégal, peu structuré, les dynamiques productives et socio-économiques qui le structure sont le lieu de la proximité, de réseaux de confiance et de coopération, de créativité, et d'apprentissage, qui sont autant de leviers des processus d'innovations pour un développement durable et inclusif (Hugon, 2014 ; Pesqueux, 2012). Ainsi comme l'affirme Pesqueux (2012), les catégories "formalisées" ne sont que des représentations de forme à un moment donné. Il n'existerait donc pas vraiment de forme, mais un continuum jamais achevé.

8 Pour toutes ces raisons, nous parlerons dans cette étude de systèmes des semences certifiées et des semences paysannes, et non de systèmes semenciers formel et informel.

\subsection{Le cadre d'analyse de la dépendance de sentier technologique}

9 En mobilisant un cadre d'analyse néo-institutionnel et évolutionniste, nous retraçons la genèse historique des mécanismes institutionnels de gouvernance des politiques semencières. En effet, les institutions sont "porteuses d'histoires" (David, 1994; Nubukpo, 2013), parce qu'elles sont encastrées (Polanyi et MacIver, 1944) dans des logiques sociales, politiques et/ou culturelles qui ne tiennent pas forcement compte du marché ou des comportements maximisateurs d'agents rationnels à une période donnée. Nous proposons d'expliquer comment se construisent sur une longue période, des attentes cohérentes mutuelles qui coordonnent les comportements des agents individuels vers une direction centralisée à l'origine "d'un paradigme dominant " ((Dosi, 1982 ; Nelson, 2009). Cela aboutirait à une dépendance de sentier, dans lequel les évènements passés continuent d'avoir une influence sur le fonctionnement présent (David, 1994 ; Sydow et al, 2012).

10 Bien que majoritairement utilisée en économie, la dépendance de sentier est aussi rencontrée dans des travaux en sciences politiques (Pierson, 2000; Thelen, 2003), pour comprendre l'évolution des technologies, des institutions, des entreprises, des stratégies. Elle est une résultante du principe de causalité cumulative de Veblen (Veblen, 1898 cité par Lazaric, 2010), qui stipule que l'accumulation d'évènements incrémentaux est à l'origine d'une trajectoire donnée dans un système ouvert et non prédéterminé. Plutôt que de considérer que l'offre et la demande sont données dans une approche déterministe, et atemporelles dans le modèle néoclassique, Veblen considère qu'elles sont le fruit d'une construction sociale. Pour comprendre cette dernière, il faut faire une "investigation génétique" pour découvrir l'origine des phénomènes observés. En outre, il faut analyser par quels mécanismes d'autorenforcement ces phénomènes s'institutionnalisent, de telle sorte que la probabilité d'être à l'état $\mathrm{i}$ au temps $\mathrm{t}+1$, est conditionnée par le fait d'avoir été à l'état $\mathrm{J}$ (incluant $\mathrm{j}=\mathrm{i}$ ) au temps $\mathrm{t}$ (David, 2007).

11 Par ailleurs, l'arrangement institutionnel choisit dans le cadre d'une dépendance de sentier n'est pas forcément optimal, mais résulte de l'histoire (Lazaric, 2010). C'est pourquoi, du fait de l'évolution des attentes de la société, cet arrangement peut à un moment donné faire l'objet de contestations, et donner lieu à une reconfiguration ou transformation du système préexistant (Mahoney, 2001). Cela crée un nouveau mécanisme institutionnel. Ces périodes annoncent la fin légale d'une période critique donnée, et sans doute le début d'une nouvelle. L'analyse économique sous l'angle de la dépendance de sentier permet donc de comprendre comment les acteurs créent des 
institutions à un moment donné critique, comment ces institutions façonnent les comportements des acteurs, et comment les réponses de ces acteurs en retour concourent au développement de nouveaux arrangements institutionnels (Mahoney, 2001).

Sydow, Schreyögg, et Koch (2005) distinguent trois étapes dans la constitution d'un sentier dépendant :

- La phase I de préformation du sentier, dans laquelle un évènement aléatoire viendrait modifier la dynamique à l'intérieur d'un système, mais sans restreindre le choix des solutions possibles. Cette phase s'achève par un moment critique. Les moments critiques sont des évènements, des particularités historiques, qui se situent en dehors des cadres théoriques disponibles, et conduisent à restreindre le champ des possibles (Mahoney, 2001)

- La phase II de formation du sentier, dans laquelle des solutions dominantes émergent et sont renforcées par les acteurs. Cette phase s'apparente à une innovation induite dans laquelle les mécanismes de renforcement sont stratégiquement manipulés par les acteurs (Garud, Kumaraswamy, et Karnoe, 2010), pour favoriser le verrouillage institutionnel. Les bénéfices relatifs (effets d'apprentissage, de coordination) à maintenir le statu quo augmente avec le temps, et augmente la probabilité de rester sur cette trajectoire (Pierson, 2000).

- La phase III de dépendance de sentier proprement dite, où l'on observe un verrouillage technologique ; les solutions sont stables et rigides.

Nous posons comme hypothèse que, dans le système semencier burkinabé, une succession d'évènements passés conduisent à une dépendance de sentier du système des semences certifiées, qui est source de verrouillage pour la diversification des trajectoires technologiques.

\section{Méthodologie}

D'un point de vue méthodologique, l'économie évolutionniste privilégie les études de cas (Beckmann et Padmanabhan, 2009 ; Bennett et Elman, 2006 ; Dobusch et Kapeller, 2013). Cette démarche consiste à analyser de manière qualitative un petit nombre d'unités (pays, entreprises, groupes, individus, régions, partis, etc). Les données sont collectées auprès de différentes sources (documents, entretiens ou enquêtes, observation participante), et le principal moyen de vérifier leur pertinence et fiabilité est la triangulation (Gerring, 2004). Cette dernière consiste à croiser ces sources pour confirmer la véracité de l'information. D'où l'intérêt d'avoir effectué une collecte de données en trois étapes.

\subsection{Un dispositif d'enquête par entretiens semi-directifs}

North (1990) propose à travers la théorie du changement institutionnel, une application aux institutions du concept de dépendance de sentier, pour expliquer la persistance de trajectoires, mais aussi les changements qui induisent des écarts de trajectoires. Le changement institutionnel est le fruit de cinq facteurs (North, 1995) : (i) l'interaction continue entre les institutions et les organisations, (ii) le capital savoir (Laperche, 2018) produit par les organisations, et qui façonne la perceptions des acteurs vis-à-vis des opportunités qui s'offrent à eux, (iii) le cadre institutionnel qui détermine ce qui est profitable ou pas (iv) les schémas mentaux des acteurs, et (v) les économies d'échelle et les externalités de réseau qui rendent le changement progressif et 
dépendant du sentier. Cette vision du changement suppose d'analyser le comportement $\mathrm{du}$ " réseau d'institutions dans les secteurs publics et privés, les activités et les interactions qui initient, modifient, importent et diffusent les nouvelles technologies " (Freeman, 1987, p.1 cité par Lazaric, 2010). Cela fait appel à la notion de Système d'Innovation (SI) qui permet de comprendre comment des organisations se structurent et se coordonnent sur un temps long, pour favoriser l'institutionnalisation de pratiques. Cela permet une meilleure adaptation sociale et culturelle de ces dernières, à l'origine d'un sentier dépendant.

Ce raisonnement nous a conduit à utiliser l'approche SI pour comprendre l'émergence et l'ancrage de cadres institutionnels de structuration du système semencier. En n'abordant pas seulement le marché, l'approche SI fait partie du courant évolutionniste et néo-institutionnelle, en ce sens qu'elle tient compte des changements cumulatifs, qui créent des mécanismes d'auto-renforcement de dynamiques institutionnelles et organisationnelles. Elle analyse l'ensemble des réseaux d'acteurs et dimensions qui concourent à la création, l'adoption, la rétention et la coordination de règles. Lesquelles règles peuvent engendrer des phénomènes d'irréversibilités, à l'origine de sentier dépendant (Philip, 1995).

17 L'approche SI conduit à identifier et enquêter les acteurs, les réseaux d'acteurs ou organisations impliqué(e)s dans l'élaboration des politiques semencières, la création variétale, la multiplication, diffusion, et commercialisation des semences. Les premières personnes interviewées (les responsables du ministère de l'agriculture) ont été identifiées à la suite d'échanges avec des chercheurs Burkinabés, partenaires de l'équipe de recherche. Par la suite, la technique «boule de neige » a été appliquée après chaque entretien. Elle consiste à partir d'un échantillon restreint pour identifier d'autres participants à l'étude au sein du cercle d'interconnaissances des personnes précédemment interviewées (Buton et al., 2016). Cette technique est particulièrement préconisée lorsqu'il n'existe pas une base de sondage des personnes appartenant à la population visée. De plus, elle reconnait la capacité des acteurs à pouvoir identifier au mieux des participants appartenant à la population cible, et avec qui ils ont des liens établis (Semaan, 2010). Cela a abouti à une catégorisation du SI en trois soussystèmes (Temple et al., 2017), représentés par la figure 1 ci-dessous.

Le sous-système recherche est constitué des acteurs dédiés à la recherche scientifique, à l'enseignement et la formation professionnelle. Il est représenté dans notre échantillon par l'Institut de l'Environnement et de Recherches Agricoles (INERA), l'université de Ouagadougou et l'Institut Supérieur des Sciences et Technologies Agricoles (ISSTA). Le sous-système intermédiation est composé d'organisations qui assurent la liaison entre la recherche et les utilisateurs, à travers le conseil, la formation, l'information, le contrôle et l'accompagnement. Il est représenté par : (i) le ministère de l'agriculture, (ii) l'Agence Nationale de Biosécurité (ANB), (iii) le Comité National des Semences (CNS), (iv) les Organisations de Producteurs (OPs), (v) les Organisations Non Gouvernementales (ONGs) et associations actives dans le secteur semencier, (vi) le projet riz pluvial, (vii) les interprofessions.

Le sous-système entrepreneurial se compose d'entreprises de multiplication des semences, des fournisseurs d'intrants et des Organisations de Producteurs (OPs). Ce système est représenté par L'Union Nationale des Producteurs de Semences du Burkina Faso (UNPSB), l'Association Nationale des Entreprises Semencières du Burkina (ANESBF), l'entreprise NAFASO (Neema Agricole du Faso), l'entreprise Bioprotect, et l'Association des Grossistes et Détaillants d'Intrants Agricoles du Burkina Faso 
(AGRODIA). L'UNPSB est une organisation professionnelle régie par la loi n014/99/AN du 15 avril 1999 portant règlementation des sociétés coopératives et des groupements au Burkina Faso. L'échantillon comporte aussi un bailleur de fonds, la FAO. Il n'est pas directement rattaché à un sous-système en particulier, puisqu'il peut intervenir de façon transversale dans chacun des sous-systèmes.

Figure 1 : Catégories d'acteurs interviewés

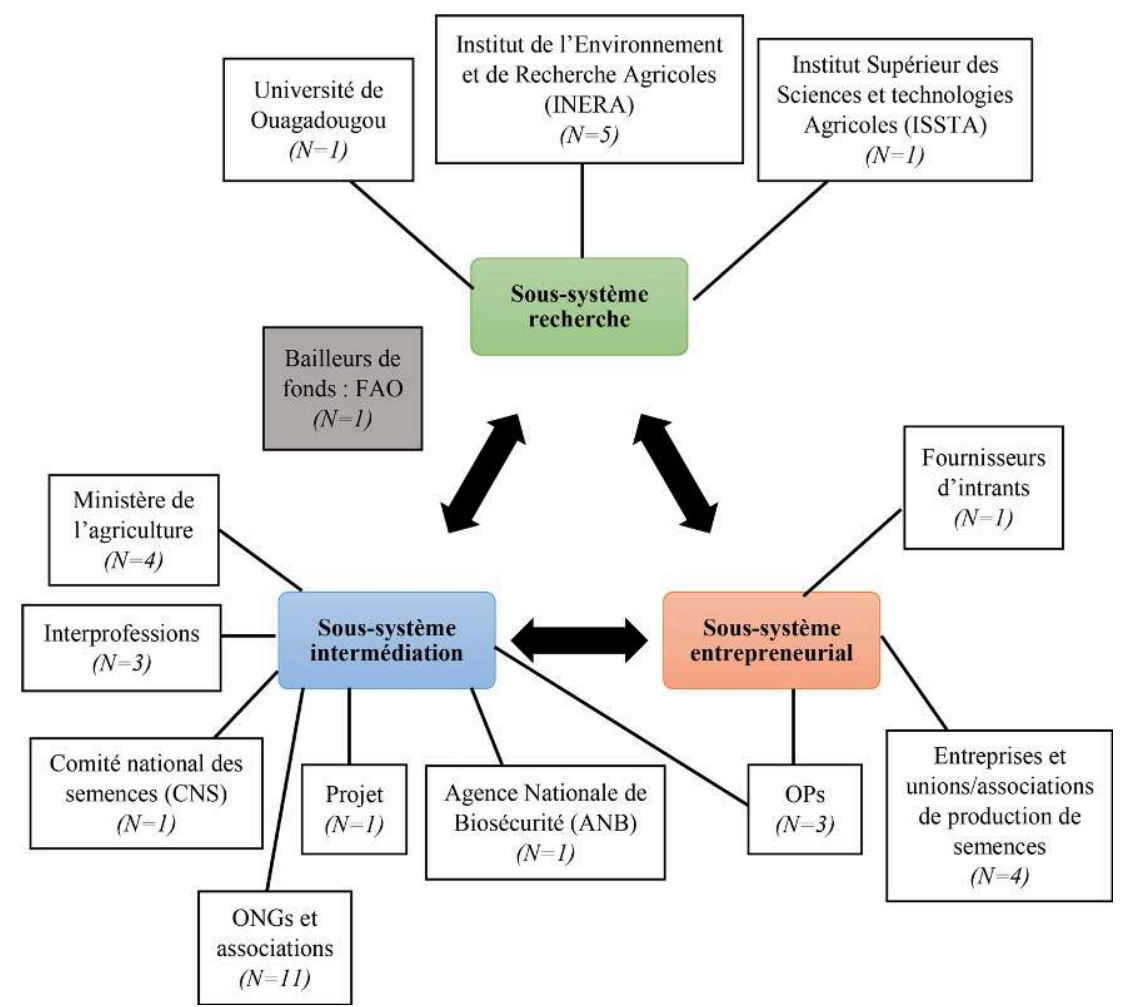

$\mathrm{N}=$ Nombre de personnes interviewées

Source: adapté de Temple et al. (2017)Au total, 37 entretiens semi-directifs ont été conduits. La liste complète des organisations interviewées est en annexe $1 \mathrm{du}$ document. Les données d'entretiens constituent le matériel chaud qu'il a fallu extraire et classer en thèmes avant de les analyser. Deux (2) thèmes ont été retenus.

Thème 1 : Réseaux d'acteurs dans le secteur semencier

Les entretiens ont permis de construire une figure des interactions dans le secteur des semences certifiées. Cette figure a été réalisée avec le logiciel $\mathrm{R}$ et met en évidence l'acteur dominant (celui qui a le plus de relations avec les autres), le rôle de chaque acteur dans le secteur semencier, et la nature des interactions entre les organisations.

\section{Thèmes 2 : Evènements déterminants du secteur semencier}

En lien avec la dépendance de sentier, nous avons mis l'accent sur les périodes qui ont contribué à structurer le secteur semencier tel qu'il se présente aujourd'hui. Ces périodes correspondent aux moments critiques (Mahoney, 2001) qui, enracinés dans des crises antérieures (guerre, conflit, catastrophe naturelle) conduisent à une sélection d'arrangements institutionnels qui renforcent une trajectoire et accroissent l'interdépendance des acteurs. Les récits des acteurs permettent aussi d'identifier les 
séquences réactives ou dites de blacklash (Mahoney, 2001) où il est possible de modifier les arrangements choisis.

Tableau 1 : Evènements déterminants du secteur semencier

\begin{tabular}{|c|c|}
\hline \multicolumn{2}{|c|}{ Evènements déterminants évoqués lors des entretiens } \\
\hline 1970-2008 & 2008-nos jours \\
\hline $\begin{array}{l}\text { «Les périodes de sècheresse ont permis au } \\
\text { gouvernement de prendre des mesures pour } \\
\text { accompagner les producteurs dans l'utilisation des } \\
\text { semences améliorées" chercheur INERA }\end{array}$ & $\begin{array}{l}\text { "Le secteur semencier a subi des bouleversements } \\
\text { surtout en 2008, avec la crise de la faim» Conseil } \\
\text { Interprofessionnel des Céréales du Burkina Faso } \\
\text { (CICB) }\end{array}$ \\
\hline $\begin{array}{l}\text { "La loi de } 2006 \text { a émis des décrets et des lois } \\
\text { d'applications aux politiques semencières» } \mathrm{CICB}\end{array}$ & $\begin{array}{l}\text { "L'année } 2008 \text { a été déterminante pour le secteur } \\
\text { semencier »Chercheur INERA }\end{array}$ \\
\hline $\begin{array}{l}\text { "La loi semencière de } 2006 \text { a bouleversé le secteur } \\
\text { semencier » FERT, NAFASO }\end{array}$ & $\begin{array}{l}\text { «A partir de 2008, l'Etat s'est vraiment investi à } \\
\text { développer le secteur semencier » Consortium riz }\end{array}$ \\
\hline & $\begin{array}{l}\text { "A partir de 2008, les subventions de l'Etat aux } \\
\text { semences certifiées ont permis de redynamiser le } \\
\text { secteur» FERT }\end{array}$ \\
\hline
\end{tabular}

Source : Auteurs à partir des verbatim d'entretiens

\subsection{Analyse documentaire}

L'analyse des documents obtenus lors des entretiens a permis de valider, invalider, nuancer et compléter les récits. Près d'une trentaine de documents ont été analysés, principalement des documents stratégiques (plans nationaux, lois/décrets, accords de partenariat, etc.) et des rapports d'études et de projets. La revue documentaire couplée aux informations issues des entretiens a permis de dresser une chronologie des évènements du secteur semencier, ainsi présentée dans le tableau 2 ci-contre.

Tableau 2 : Chronologie des évènements du secteur semencier

\begin{tabular}{|l|l|l|}
\hline \multicolumn{2}{|c|}{$1970-2008$} \\
\hline Dates & \multicolumn{1}{|c|}{ Evènements/activités } & \multicolumn{1}{|c|}{ Sources } \\
\hline 1970 & Sècheresse & Entretiens \\
\hline 1974 & Projet de multiplication des semences financé par l'USAID & $\begin{array}{l}\text { The African Trade } \\
\text { investment program (2002) } \\
\text { Ministère de l'Agriculture, } \\
\text { l'Hydraulique et des } \\
\text { Ressources Halieutiques } \\
(2011)\end{array}$ \\
\hline
\end{tabular}




\begin{tabular}{|c|c|c|}
\hline 1984 & Programme de recherche sur le riz et la riziculture & - INERA (1998) \\
\hline 1988 & $\begin{array}{l}\text { Appui CEDEAO/FAO/PNUD/FENU à l'approvisionnement, le } \\
\text { contrôle de qualité, la formation en matière de semences }\end{array}$ & \multirow{2}{*}{$\begin{array}{l}\text { - Ministère de l'Agriculture, } \\
\text { de l'Hydraulique et des } \\
\text { Ressources } \text { Halieutiques } \\
(2011)\end{array}$} \\
\hline 1990 & $\begin{array}{l}\text { Appui aux fermes semencières, avec notamment la mise en } \\
\text { place de la ferme semencière de Bani et le réseau de } \\
\text { multiplication et de diffusion des semences améliorées dans } \\
\text { la région du Sahel }\end{array}$ & \\
\hline 1993 & $\begin{array}{l}\text { Soutien FAO et PNUD à l'élaboration du programme de } \\
\text { développement du secteur semencier }\end{array}$ & $\begin{array}{l}\text { - Ministère de L'agriculture } \\
\text { et de la Sécurité Alimentaire } \\
(2013)\end{array}$ \\
\hline 1996 & $\begin{array}{l}\text { Début des activités de préservation des semences paysannes } \\
\text { de la FENOP }\end{array}$ & Entretien FENOP \\
\hline 1998 & $\begin{array}{l}\text { Projet « survival » de préservation des semences paysannes } \\
\text { conduit par l'APN Sahel et financé par l'USC canada }\end{array}$ & Entretien APN Sahel \\
\hline 2000 & $\begin{array}{l}\text { Création de AFSTA (African Seed Trade Association) au niveau } \\
\text { sous régional }\end{array}$ & $\begin{array}{l}\text { - } \quad \text { African Centre for } \\
\text { biodiversity (2015) }\end{array}$ \\
\hline 2002 & Création d'une spécialisation semences à Matroukou & $\begin{array}{l}\text { Entretien avec le Service } \\
\text { National des Semences (SNS) }\end{array}$ \\
\hline \multirow{2}{*}{2003} & $\begin{array}{l}\text { Projet de développement du secteur semencier avec l'appui } \\
\text { de la JICA }\end{array}$ & \multirow{2}{*}{$\begin{array}{l}\text { Entretiens avec CICB, FERT, } \\
\text { NAFASO, UNPSB, SNS }\end{array}$} \\
\hline & $\begin{array}{l}\text { Création de l'Union Nationale de Producteurs semenciers du } \\
\text { Burkina Faso (UNPSB), mais reconnue officiellement en } 2004\end{array}$ & \\
\hline \multirow{4}{*}{2006} & Validation à Lomé de la politique semencière sous régionale & \multirow{4}{*}{$\begin{array}{l}\text { - CORAF, CEDEAO, \& USAID } \\
(2014) \\
\text { - République du Burkina Faso } \\
(2006) \\
\text { - African Centre for } \\
\text { biodiversity (2015) }\end{array}$} \\
\hline & Loi semencière & \\
\hline & Création de l'AGRA (Alliance for a Green Revolution in Africa) & \\
\hline & Première loi sur le régime en matière de biosécurité & \\
\hline & Activités en lien avec les semences améliorées/certifiées & \\
\hline & Activités de promotion des semences paysannes & \\
\hline
\end{tabular}

\begin{tabular}{|l|l|l|}
\hline \multicolumn{2}{|c|}{$2008-$ nos jours } \\
\hline Dates & Evènements/activités & Sources \\
\hline
\end{tabular}




\begin{tabular}{|c|c|c|}
\hline \multirow{4}{*}{2008} & Achat régulier des semences par l'Etat & \multirow{4}{*}{$\begin{array}{l}\text { - Ouedraogo (2011); MASA et FAO } \\
(2014) \text {; } \\
\text { - Holtzman, Kaboré, Tassembedo, et } \\
\text { Adomayakpor (2013) } \\
\text { - SPAAA et FAO (2013); FAO (2014) } \\
\text { - Entretiens UNPSB, INERA } \\
\text { - Réseau mondial pour le droit à } \\
\text { l'alimentation et à la nutrition et } \\
\text { Convergence globale des luttes pour la } \\
\text { terre et l'eau (2018) }\end{array}$} \\
\hline & $\begin{array}{l}\text { Accroissement du budget de l'Etat dans le secteur } \\
\text { semencier }\end{array}$ & \\
\hline & $\begin{array}{l}\text { Projet de Développement des Semences } \\
\text { Améliorées (PDSA) financé par la JICA }\end{array}$ & \\
\hline & $\begin{array}{l}\text { Divers appuis FAO, JICA, GIZ au secteur semencier } \\
\text { (formation, achat et redistribution de semences } \\
\text { améliorées, équipements de laboratoires) }\end{array}$ & \\
\hline 2009 & $\begin{array}{l}\text { Création de la } \\
\text { gestion des ressources phytogénétiques } \\
\text { (CONAGREP) }\end{array}$ & - République du Burkina Faso (2009) \\
\hline \multirow{2}{*}{2011} & $\begin{array}{l}\text { Stratégie de développement durable du secteur } \\
\text { semencier }\end{array}$ & $\begin{array}{l}\text { - Ministère de l'agriculture et des } \\
\text { aménagements hydrauliques (2010) }\end{array}$ \\
\hline & $\begin{array}{l}\text { Programme de productivité en Afrique de l'Ouest } \\
\text { (PPAAO) }\end{array}$ & \\
\hline 2011 & $\begin{array}{l}\text { Projet de renforcement des systèmes semenciers } \\
\text { traditionnels }\end{array}$ & - INERA (2014) \\
\hline \multirow{3}{*}{2012} & Programme National du Secteur Rural (PNSR I) & \multirow{3}{*}{ - République du Burkina Faso (2012) } \\
\hline & Programme semencier ouest africain (PSAO) & \\
\hline & $\begin{array}{l}\text { Promulgation d'une nouvelle loi sur la } \\
\text { biotechnologie et abrogation de la précédente }\end{array}$ & \\
\hline \multirow{2}{*}{2013} & $\begin{array}{l}\text { Adoption d'un cadre règlementaire sous régionale } \\
\text { sur les semences }\end{array}$ & \multirow{2}{*}{$\begin{array}{l}\text { - CEDEAO, CILSS, et UEMOA, (2015) } \\
\text { - Djamen (2016) }\end{array}$} \\
\hline & $\begin{array}{l}\text { Mise en place de l'alliance pour l'industrie } \\
\text { semencière en Afrique de l'Ouest }\end{array}$ & \\
\hline 2014 & $\begin{array}{l}\text { Elaboration du catalogue national des espèces et } \\
\text { plants }\end{array}$ & - Comité National des Semences (2014) \\
\hline \multirow[b]{2}{*}{2014} & Création du Collectif citoyen pour l'agroécologie & \multirow{2}{*}{$\begin{array}{l}\text { - Entretien avec porte-parole CCAE, } \\
\text { INERA, Inades formation } \\
\text { - GFA Consulting Group (2016) }\end{array}$} \\
\hline & $\begin{array}{l}\text { Engouement de la société civile à la préservation } \\
\text { des semences paysannes }\end{array}$ & \\
\hline 2015 & $\begin{array}{l}\text { Réalisation d'un documentaire «Et si le tô venait } \\
\text { à disparaitre?» }\end{array}$ & Entretien, revue de presse \\
\hline 2016 & $\begin{array}{l}\text { Plaidoyer pour la prise en compte de } \\
\text { l'agroécologie dans le PNSR2 }\end{array}$ & Confédération Paysanne du Faso (2016) \\
\hline
\end{tabular}




\begin{tabular}{|c|c|c|}
\hline \multirow{3}{*}{2017} & $\begin{array}{l}\text { Deuxième programme national du secteur rural } \\
\text { (PNSR) }\end{array}$ & \multirow{3}{*}{$\begin{array}{l}\text { - République du Burkina Faso (2017) } \\
\text { - Entretiens NAFASO, UNPSB, CNS } \\
\text { - GFA Consulting Group (2016) } \\
\text { - CEDEAO (2017) }\end{array}$} \\
\hline & $\begin{array}{l}\text { Création de l'association nationale des } \\
\text { entreprises semencières du Burkina Faso (ANESB- } \\
\text { F) }\end{array}$ & \\
\hline & $\begin{array}{l}\text { Système semencier classé comme le plus } \\
\text { performant en Afrique de l'Ouest }\end{array}$ & \\
\hline \multirow{2}{*}{2017} & $\begin{array}{l}\text { Projet de loi pour la préservation des ressources } \\
\text { phytogénétiques }\end{array}$ & \multirow{2}{*}{$\begin{array}{l}\text { - Entretien CONAGREP, COASP, ANSD, } \\
\text { point focal agroécologie }\end{array}$} \\
\hline & $\begin{array}{l}\text { Elaboration d'un catalogue des semences } \\
\text { paysannes dans l'Est du Burkina Faso }\end{array}$ & \\
\hline 2018 & $\begin{array}{l}\text { Processus de commercialisation des semences } \\
\text { dans l'espace OCDE en cours }\end{array}$ & - MAAH, GIZ, et GNIS (2018) \\
\hline 2018 & $\begin{array}{l}\text { Réalisation du documentaire Burkinabé Bounty sur } \\
\text { l'agroécologie }\end{array}$ & Revue de presse ${ }^{1}$ \\
\hline & \multicolumn{2}{|l|}{ Activités en lien avec les semences améliorées/certifiées } \\
\hline & on des semences paysannes & \\
\hline
\end{tabular}

\subsection{Forum multi-acteurs}

Le forum complète la démarche constructiviste ${ }^{2}$ apportée à cette étude, en revêtant deux objectifs : produire de la connaissance de façon collective sur les phénomènes observés, et accompagner les acteurs dans une intention délibérée de changement ou de transformation (Krief et Zardet, 2013). Ils permettent de mettre en place un effet miroir (Krief et Zardet, 2013) dont le but est de valider, invalider, enrichir, nuancer les résultats. L'effet miroir n'a pas vocation à compter la quantité de mots ou idées émises par les acteurs, mais plutôt de restituer la diversité des points de vue, sans juger, ni interpréter. En faisant interagir les acteurs entre eux, mais aussi avec les chercheurs, les forums reposent sur un processus concomitant d'harmonisation des connaissances sur le secteur semencier, et de mise en discussion des conditions d'émergence de nouveaux arrangements institutionnels. Selon Callon et Barthe (2005), les forums multi-acteurs ou «hybrides ", mettent en avant le concept de "rationalité-négocié ", qui suppose un processus de négociations et de délibérations entre scientifiques, décideurs politiques et profanes.

Nous avons mobilisé cette démarche, en co-organisant un forum au Burkina Faso. Pour ce faire, nous avons contacté par mail et par appel téléphonique toutes les personnes précédemment interviewées (section 3.1), pour recenser leurs disponibilités à 
participer au forum. Il leur était aussi demandé de faire passer le message autour d'eux auprès d'autres acteurs du secteur semencier, que nous n'aurions pas enquêtés par manque de temps. L'objectif étant de faire ressortir une diversité de points de vue sur l'évolution du secteur des semences certifiées, et discuter de ses modalités de coexistence avec le secteur des semences paysannes. La période de décembre 2018 fut retenue par la plupart des participants disponibles. Trente-trois (33) personnes furent présentes au forum dont 2 représentants du secteur public, 12 chercheurs, 2 acteurs $d u$ secteur privé (production de semences), et 17 représentants d'OPs et autres organisations de la société civile.

Le forum a mis en évidence l'interdépendance entre les acteurs des semences certifiées et des semences paysannes, et les blocages de chaque système à l'épanouissement de l'autre. Le rapport du forum validé de façon participative par les participants a conduit à l'élaboration d'un policy brief (Sawadogo et al. 2020), qui propose divers axes d'interventions pour renforcer le système semencier dans son ensemble. Ces axes comprennent entre autres : (i) l'accompagnement des producteurs à la caractérisation des semences paysannes d'intérêt général, (ii) l'appui à la mise en place de banques de gènes pour la conservation des semences, (iii) la transparence et l'explicitation dans la formation du prix des semences améliorées, qui reste un déterminant majeur de non adoption pour les producteurs, (iv) le renforcement du système de certification pour réduire au maximum les fraudes.

\section{Résultats et discussion}

L'analyse par triangulation des informations issues des entretiens, de la revue documentaire et $\mathrm{du}$ forum met en exergue deux périodes critiques du secteur semencier burkinabé. La première qui part de la sècheresse des années 70 à la crise de la faim en 2008, a donné lieu à une transposition des cadres de régulation du système semencier des pays industriels au Burkina Faso (phénomène de déplacement institutionnel). Ces nouvelles règles rencontrent celles ancestrales d'échanges de semences et de sélection massale, et cela abouti à une juxtaposition de règles. Dans la seconde période (2008 - 2019), pendant que la reproduction institutionnelle du système des semences certifiées s'intensifie, des séquences réactives de préservation des semences paysannes, jusque-là restreintes à l'échelle locale, s'étendent au niveau national pour favoriser la reconfiguration du système semencier dans son ensemble.

\subsection{0 - 2008 : Déplacement et sédimentation institutionnels}

La question des semences commence à se poser au Burkina Faso dans les années 70, à la suite d'une grande sécheresse qui aura marqué le pays et le monde rural en particulier. En 1974, l'Etat crée le Service National des Semences (SNS) et affirme ainsi sa volonté d'approvisionner les producteurs en semences de qualité précoces, à hauts rendements (Ouedraogo, 2011). Avec l'aide de l'USAID (United States Agency for International Development), de la CEDEAO (Communauté Economique des Etats de l'Afrique de l'Ouest), de la FAO (Food and Agriculture Organization), du Programme des Nations Unies pour le Développement (PNUD) et du Fonds d'Equipement des Nations Unies (FENU), l'Etat burkinabé met sur pied un projet de multiplication de semences, avec pour objectif d'approvisionner les producteurs en semences de qualité. 

providence de Keynes (James, 1955 ; Frydman, 1988) pour relancer les économies meurtries par une crise qui amplifie la rareté des ressources. Pour Keynes, l'intervention de l'Etat est en effet souhaitable dans les situations de déficiences des marchés, à travers des allocations ou des politiques de relance pour stimuler un secteur de l'économie inexistant ou défaillant. C'est ainsi qu'un régime budgétaire d'expansion (Koussoubé et al., 2015) s'est mis en place avec l'idée que le développement rime avec croissance économique, accumulation de ressources. Mais cela conduira à la fin des années 80 à un endettement progressif de l'Etat, du fait de coûts de fonctionnement élevés (Sanfo, 2010). A partir du début des années 90, le Fonds Monétaire International (FMI), soutenu par les économistes libéraux, va imposer une réduction drastique des dépenses de l'Etat notamment dans l'agriculture. La notion de développement est dès lors associée à la bonne gouvernance pour permettre aux Etats de mieux gérer leurs ressources et de ne pas sombrer dans le surendettement. Les Programmes d'Ajustements Structurels (PAS) naissent dans cette dynamique, et s'imposent à de nombreux pays africains comme la solution idoine pour un progrès durable.

Malgré ces mesures restrictives, l'Etat burkinabé soutenu par quelques institutions internationales (FAO, PNUD, JICA) continue sa mission de promotion et de structuration du système semencier conformément à la politique nationale semencière qui prévalait en ce temps. En 1993, il élabore le programme de développement du secteur semencier dont l'objectif est de "non seulement fournir à un prix acceptable et en quantité suffisante des semences de qualité, provenant de variétés améliorées bien adaptées à l'écologie et à la technologie locales, mais aussi inciter le monde rural à une utilisation accrue de ces articles de sélection" (Ministère de l'agriculture et des ressources animales et FAO, 1992, p. 37). Il créé en 2002 une spécialisation "semences » à l'école technique et professionnelle des techniciens semenciers à Mantouroukou pour renforcer les capacités des cadres du SNS. En 2004, il appuie la création, l'organisation et la structuration de l'Union Nationale des Producteurs Semenciers du Burkina Faso (UNPSB), dont l'objectif est de promouvoir et produire des semences certifiées issues de la recherche. L'idée est que le fait d'appartenir à un groupe accroit la confiance, la circulation de l'information, et génèrent des effets positifs sur l'usage de la technologie

L'année 2006 marque un tournant décisif dans le secteur semencier, à travers la promulgation de la loi semencière. Cette dernière donne un cadre règlementaire pour la production et la commercialisation des semences certifiées et inscrites au catalogue national des espèces et des plants. Seules les semences dites " améliorées ", protégées par des droits de propriété intellectuelle et répondant aux critères DHS (Distinction, homogénéité, stabilité) peuvent être produites et commercialisées ; aucune mention n'est faite à ce sujet sur les semences traditionnelles/paysannes. Elle consacre aussi une section à la protection des ressources phytogénétiques traditionnelles qui doit être assurée par l'Etat dans l'intérêt de la nation (République du Burkina Faso, 2006). C'est cette loi qui crée la scission entre les semences dites améliorées (et donc plus intéressantes du point de vue agronomique), et les autres connues sous le vocable de semences paysannes, traditionnelles.

Dans cette phase de préformation du chemin de sentier, le régime s'oriente vers un déplacement institutionnel (Mahoney and Thelen, 2010) dans lequel, les arrangements traditionnels de partage et d'échange de semences entre producteurs sont officiellement remplacées par des règles nouvelles. En effet, avant l'arrivée des normes 
de régulations formelles du secteur semencier, les règles coutumières (non formalisées) ont consisté à échanger des semences pendant des années ou à ressemer la même semence pour maintenir la diversité nécessaire à l'adaptation aux changements environnementaux (Ilbert et Louafi, 2004). Soutenu à partir des années 90 par les OPs et autres organisations de la société civile (ONGs) qui brandissent les notions d "empowerment ", "capabilities", le système semencier paysan de préservation et de promotion des semences paysannes a continué d'évoluer dans un contexte qui ne tient compte que des logiques paysannes endogènes et socialement encastrées (Polanyi et MacIver, 1944). Maggetti (2014) affirme que le déplacement institutionnel peut être endogène ou issu de l'importation de modèles étrangers. Ce dernier cas caractérise la réglementation semencière burkinabé calquée sur le modèle européen de l'UPOV (Union Internationale pour la Protection des Obtentions Végétales) (Coulibaly et Brac de la Perrière, 2019).

Bien que dans les textes officiels on voit émerger un modèle institutionnel de déplacement, dans la mise en œuvre pratique, il s'agit d'une sédimentation institutionnelle (Thelen, 2003). Cette dernière se caractérise par le fait d'ajouter de nouvelles institutions (loi semencière) sans démanteler les anciennes (sélection massale et troc de semences); ce qui aboutit à un mélange ou une coexistence d'anciennes et de nouvelles règles. L'évolution des cadres institutionnels ne s'accompagne pas toujours d'un changement des fondements de l'action individuelle, ni d'une adaptation de tous les acteurs à la nouvelle institution sous la forme de «reproduction institutionnelle » (Thelen, 2003). Ainsi, bien que la règlementation mette l'emphase sur la commercialisation et l'usage des semences certifiées, les producteurs ont continué d'utiliser leurs semences paysannes ; ce qui a maintenu entre 1970 et 2008, le taux d'utilisation des semences certifiées céréalières à un niveau relativement bas (6-7\%) (MASA et FAO, 2014 ; Kaboré, Dabat, et Vom Brocke, 2008). Comme l'affirme Ansaloni (2017, p. 100), « instituer un marché, ou des mécanismes marchands, ne permet pas de gouverner à distance les comportements des individus, en quelque sorte de manière mécanique».

\subsection{8 à nos jours : Dépendance de sentier et séquences réactives}

\subsubsection{La dépendance de sentier}

Les entretiens ont révélé qu'à partir de la crise alimentaire de 2008, le gouvernement burkinabé a réaffirmé sa volonté à promouvoir l'utilisation des variétés améliorées à haut rendement. C'est la phase de formation du sentier, qui se matérialise par l'accumulation d'actifs spécifiques (infrastructures et compétences), et d'actifs secondaires immatériels (création de réseaux, orientations politiques) (Casadella et Tahi, 2017). Cette phase se caractérise aussi par une forte interdépendance des acteurs, et des externalités positives (accroissement des parts de marchés, développement d'un secteur privé d'approvisionnement en intrants) qui renforcent la trajectoire du système des semences certifiées (Sydow et al., 2012).

\section{a. Accumulation d'actifs}


Tableau 3 : Actifs de caractérisation de la dépendance de sentier

\begin{tabular}{|c|c|c|}
\hline Types d'actifs & Désignation & $\begin{array}{l}\text { Sources de } \\
\text { financement }\end{array}$ \\
\hline Infrastructures & Construction de 4 laboratoires du SNS & FAO \\
\hline \multirow{4}{*}{ Compétences } & $\begin{array}{l}\text { Projet de développement des semences } \\
\text { améliorées (PDSA) }\end{array}$ & JICA \\
\hline & $\begin{array}{l}\text { Programme de productivité Agricole en Afrique } \\
\text { de l'Ouest (PPAAO) }\end{array}$ & Banque mondiale \\
\hline & $\begin{array}{l}\text { Programme semencier en Afrique de l'Ouest } \\
\text { (PSAO) }\end{array}$ & USAID \\
\hline & $\begin{array}{l}\text { Formation des cadres du SNS par les agents de } \\
\text { l'INERA }\end{array}$ & Etat burkinabé \\
\hline \multirow{5}{*}{ Création de réseaux } & $\begin{array}{l}\text { Association Nationale des } \quad \text { Entreprises } \\
\text { semencières (ANESBF) }\end{array}$ & $\begin{array}{l}\text { Initiative privée des } \\
\text { entreprises }\end{array}$ \\
\hline & $\begin{array}{l}\text { Alliance pour la révolution verte en Afrique } \\
\text { (AGRA) }\end{array}$ & $\begin{array}{l}\text { Fondations Bill et } \\
\text { Melinda Gates, } \\
\text { Rockfeller }\end{array}$ \\
\hline & $\begin{array}{l}\text { Forum sur les biotechnologies agricoles au } \\
\text { Burkina }\end{array}$ & AGRA \\
\hline & $\begin{array}{l}\text { Alliance pour une industrie semencière en } \\
\text { Afrique de l'Ouest (ASIWA) }\end{array}$ & USAID \\
\hline & $\begin{array}{l}\text { Nouvelle alliance pour la sécurité alimentaire et } \\
\text { la nutrition (NASAN) }\end{array}$ & G7 \\
\hline \multirow{5}{*}{$\begin{array}{l}\text { Politiques } \\
\text { documents } \\
\text { stratégiques }\end{array}$} & Politique de subvention aux intrants & $\begin{array}{l}\text { Etat burkinabé, FAO, } \\
\text { GIZ }\end{array}$ \\
\hline & Catalogue nationale des espèces et des plants & FAO \\
\hline & Programmes Nationaux du Secteur Rural (I et II) & Etat burkinabé \\
\hline & Loi semencière sous-régionale & CEDEAO \\
\hline & $\begin{array}{l}\text { Politique sous-régionale facilitant le commerce } \\
\text { des semences entre les Etats de l'espace CEDEAO- } \\
\text { UEMOE-CILSS }\end{array}$ & CEDEAO, UEMOA, CILSS \\
\hline
\end{tabular}

Avec l'appui de partenaires techniques et financiers, le Burkina a au fil du temps mis en place des mesures destinées à promouvoir la production à grande échelle des semences certifiées, et leur utilisation. L'une des orientations phares est la politique de subvention aux intrants qui crée des pour et des contre dans la société. 
Figure 2: Répartition moyenne du budget de l'Etat au secteur agricole entre 2006 et 2013

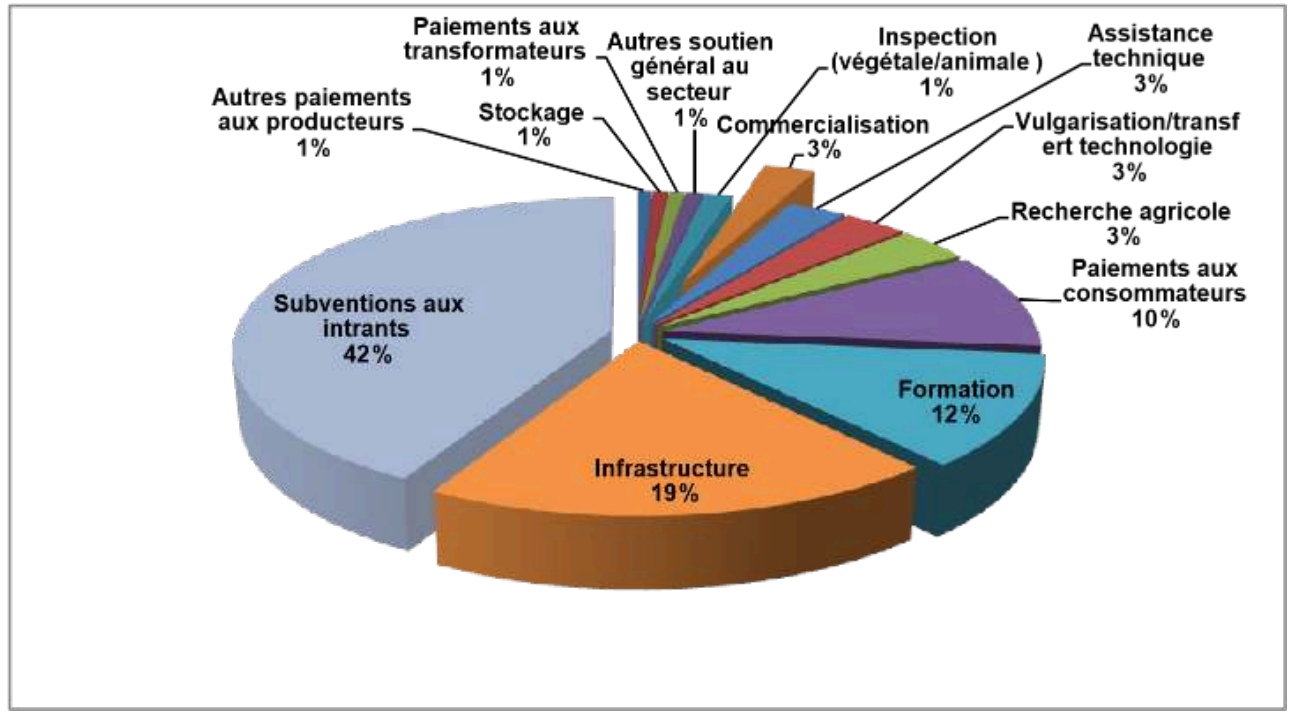

Source : $(F A O, 2014)$

Figure 3: Evolution du nombre de producteurs de semences

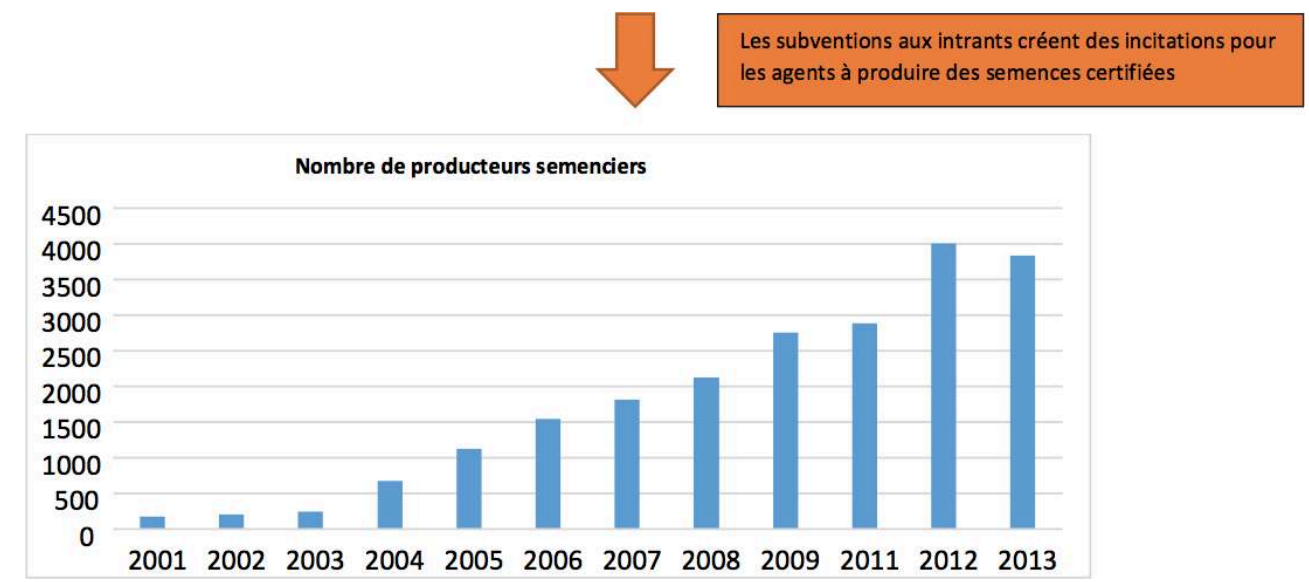

Source : Auteurs à partir des données du MASA (2013) et CORAF, CEDEAO, \& USAID (2014) 


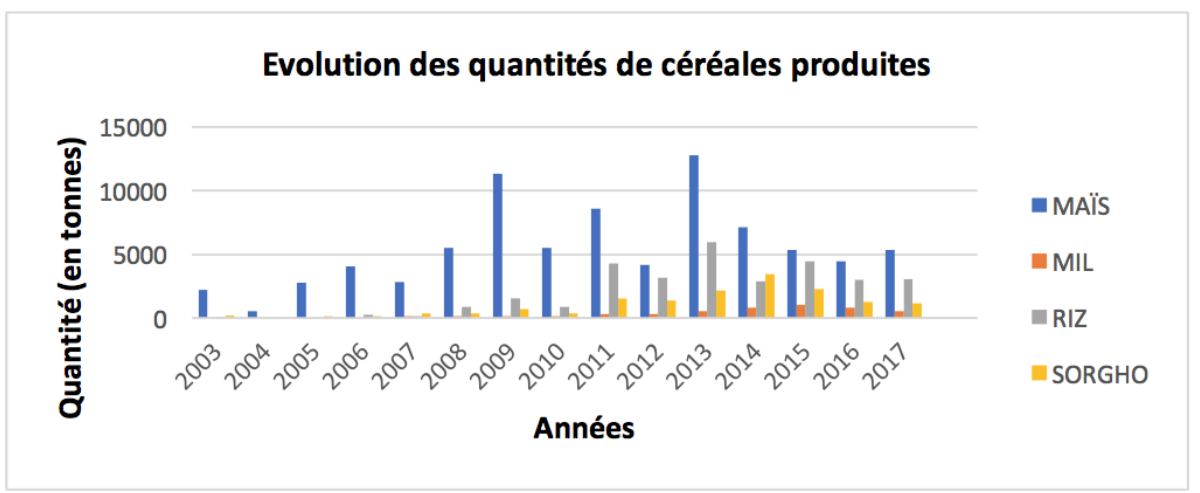

Source : Auteurs à partir des données du Service National des Semences (SNS) et du rapport du MASA (2013)

L'analyse de l'évolution des dépenses publiques entre 2006-2013, montre une tendance relativement croissante, et un intérêt particulier pour la subvention aux intrants qui occupe progressivement une part importante du budget. Chaque année, l'Etat achète des semences aux producteurs de semences rassemblés autour de l'Union Nationale de producteurs semenciers (UNPSB), et de l'Association Nationale des Entreprises Semencières (ANESBF), et les redistribue à prix subventionnés aux populations «vulnérables ». L'UNPSB étant plus ancienne (créée en 2004) que l'ANESBF qui date 2017 , elle livre $80 \%$ de la demande nationale, contre $20 \%$ pour l'ANESBF. Cette période est marquée par le développement d'une nouvelle forme d'agro business, la production de semences améliorées comme véritable source de revenus, et la diversification de la production agricole. Entre 2001 et 2013, le nombre de producteurs semenciers a considérablement augmenté passant de 175 en 2001 à 3827 en 2013 (MASA, 2013). Les personnes interviewées ont précisé que pendant longtemps, toutes (une grande partie) les actions de l'Etat étaient orientées vers le développement de la culture du coton, principale source de revenu agricole. Mais à partir de 2008, notamment suite à l'élaboration du premier Programme National du Secteur Rural (PNSR I) en 2011, le gouvernement Burkinabé reconnait l'intérêt des autres cultures non seulement dans la génération de revenus, mais aussi dans l'atteinte de la sécurité alimentaire des ménages.

Cependant, les subventions de l'Etat aux semences certifiées sont contestées par les membres de l'association des négociants d'agro-intrants (AGRODIA). Ces derniers estiment que ce programme freine le développement de leurs propres réseaux de distributions, et crée de ce fait des distorsions économiques. Pour eux, le programme n'encourage pas les producteurs à se rapprocher des producteurs de semences, et les plongent dans une logique attentiste, alors que tout le monde ne peut bénéficier de ces semences. En effet, selon les responsables du ministère de l'agriculture, «les subventions de l'Etat ne représenteraient que $5 \%$ des besoins en semences des producteurs ». Or, d'après une étude conduite en 2014 par la FAO et le Ministère de l'Agriculture, 69\% des producteurs obtiennent leurs semences de structures étatiques. Cela est dû non 
seulement au programme de subvention des intrants, mais aussi à l'implication de nombreux projets dans la distribution des semences (MASA et FAO, 2014)

Figure 5: Source d'approvisionnement en semences certifiées

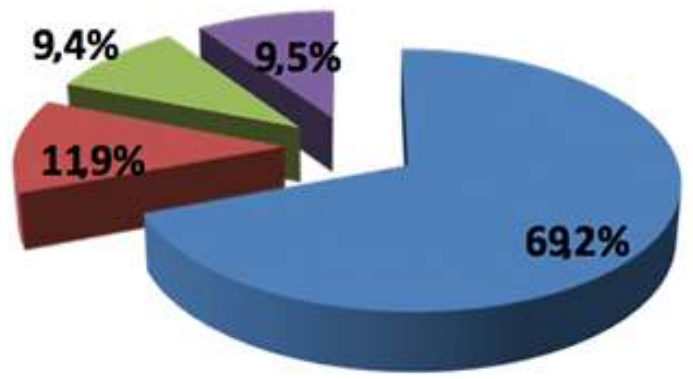

Structure de l'Etat - ONG " Producteurs semenciers Revendeurs

Source : (MASA \& FAO, 2014) persistance structurelle dans le système formel à travers la création d'un secteur privé fort structuré majoritairement autour de l'UNPSB et de l'ANESBF. L'UNPSB compte aujourd'hui près de 3000 producteurs rassemblés autour des groupements de producteurs, d'unions départementales, de 45 unions provinciales, et de 13 unions régionales. Sa renommée et les partenariats tissés avec le ministère de l'agriculture, la recherche, et les projets/programmes nationaux et sous régionaux, lui permettent d'obtenir des parts de marchés aussi bien au Burkina Faso, que dans d'autres pays de la sous-région (Sénégal, Côte d'Ivoire, Sierra Léone, Libéria, Guinée et Nigéria). D’après les données mobilisables à l'UNPSB, les marchés acquis par l'union représentaient environ 7 milliards de FCFA en 2014, 5,6 milliards en 2015 et 1,6 milliards en 2016. pied d'outils de financement agricole. En 2018, l'Etat burkinabé a obtenu l'agrément de la commission bancaire de l'Union Economique et Monétaire Ouest-Africaine (UEMOA) pour la création d'une banque agricole. Son actionnariat est composé de 13 Organisations Paysannes (OP), 3 sociétés d'Etat, 8 sociétés privées et 70 personnes physiques. Dans son conseil d'administration, les OPs seront représentées par la 
Confédération paysanne du Faso (CPF), l'Union Nationale des Producteurs de Coton (UNPCB), et l'UNPSB (Confédération Paysanne du Faso, 2017).

42 Le fait que l'Etat soit le plus grand acheteur de semences favorise la promotion de systèmes rentiers tirés par les marchés intérieurs (Magrin, 2015). En effet, plutôt que de dépendre de l'extérieur pour commercialiser leurs semences, comme c'est souvent le cas des matières premières en Afrique, les acteurs du système semencier génèrent des revenus principalement grâce aux achats de l'Etat. Kpadé et Boinon (2011) ont mis en évidence le rôle d'une politique de libéralisation dans la dynamique de création et d'innovation institutionnelles. Même s'il ne s'agit là que d'une libéralisation partielle (l'approvisionnement en intrants) parce que la création variétale et la certification restent l'apanage du secteur public, l'implication des acteurs privés dans les processus de négociations politiques renforce la formation du sentier dépendant.

La mobilisation et les investissements consentis dans la promotion des semences améliorées font du Burkina en 2017, le pays de la sous-région le plus avancé ${ }^{3}$ dans l'application des normes semencières sous régionales. La reproduction institutionnelle s'intensifie avec le démarrage en 2018 du processus d'adhésion aux systèmes de semences de l'OCDE, de l'UE et à l'accréditation aux normes de l'ISTA (International Seed testing Association) ${ }^{4}$. Ce processus implique un audit des infrastructures de création variétale, de certification et de production des semences afin de les mettre aux normes ISTA. Le Burkina est appuyé dans cette démarche par le Groupement National Interprofessionnel des Semences et Plants de la France (GNIS), et le Projet Centre d'Innovation Vertes (ProCIV) du ministère de la coopération allemande. Cette initiative permettra au pays d'échanger des semences avec les 60 autres pays membres du schéma de certification OCDE.

\section{c. Des relations fortes entre les acteurs}

En lien avec la logique de la révolution verte, l'importance accordée aux semences certifiées est le fruit de l'interaction entre des cadres et des acteurs à l'échelle national, sous régional et international. Cela aboutit à une configuration d'acteurs (figure 6) dans laquelle la recherche publique représentée par l'INERA demeure l'acteur central, du fait des différentes fonctions qui lui sont assignées. L'INERA détient le monopole de la création variétale et collabore avec d'autres organismes de recherche tels que le CIRAD (Centre de Coopération Internationale en Recherche Agronomique pour le Développement), l'ICRISAT (International Crops Research Institute for the Semi-Arid Tropics), Africa Rice. Il produit et commercialise les semences de base dans ses différentes stations, mais aussi sous contrat avec des producteurs ou entreprises individuel(le)s de semences. L'INERA intervient aussi dans la formation et l'accompagnement des producteurs à l'utilisation des résultats de la recherche. Il forme les agents du SNS, et est impliqué dans la définition des politiques du secteur semencier. Il est de ce fait membre du Comité National des semences (CNS), et de la Commission Nationale de Préservation des Ressources Phytogénétiques (CONAGREP).

La seconde catégorie d'acteurs fortement représentée dans le système semencier est le secteur privé composé par l'UNPSB, l'entreprise NAFASO (leader dans la production des semences), l'ANESB-F, les producteurs, les distributeurs d'intrants tels que AGRODIA, Agrisahel, COCIMA (Coopérative de Commercialisation d'Intrants et de Matériels Agricoles). Le secteur privé intervient dans la production, la commercialisation et l'usage des semences améliorées. 
Son essor est soutenu par la troisième catégorie d'acteurs que sont les services publics (ministères de l'agriculture et du commerce), à travers l'achat régulier des semences, la délivrance d'agrément de commercialisation et la régulation du secteur. Zheng et al., (2015) ont mis en évidence l'importance des liens entre les organisations politiques et les entreprises pour favoriser le développement des affaires. Dans le secteur semencier, cela aura contribué à consolider la trajectoire du système formel à travers l'octroi de ressources aux entreprises (formation, achat de semences), qui a pour corollaire l'augmentation des quantités de semences certifiées.

Figure 6: Interactions des acteurs du système des semences certifiées

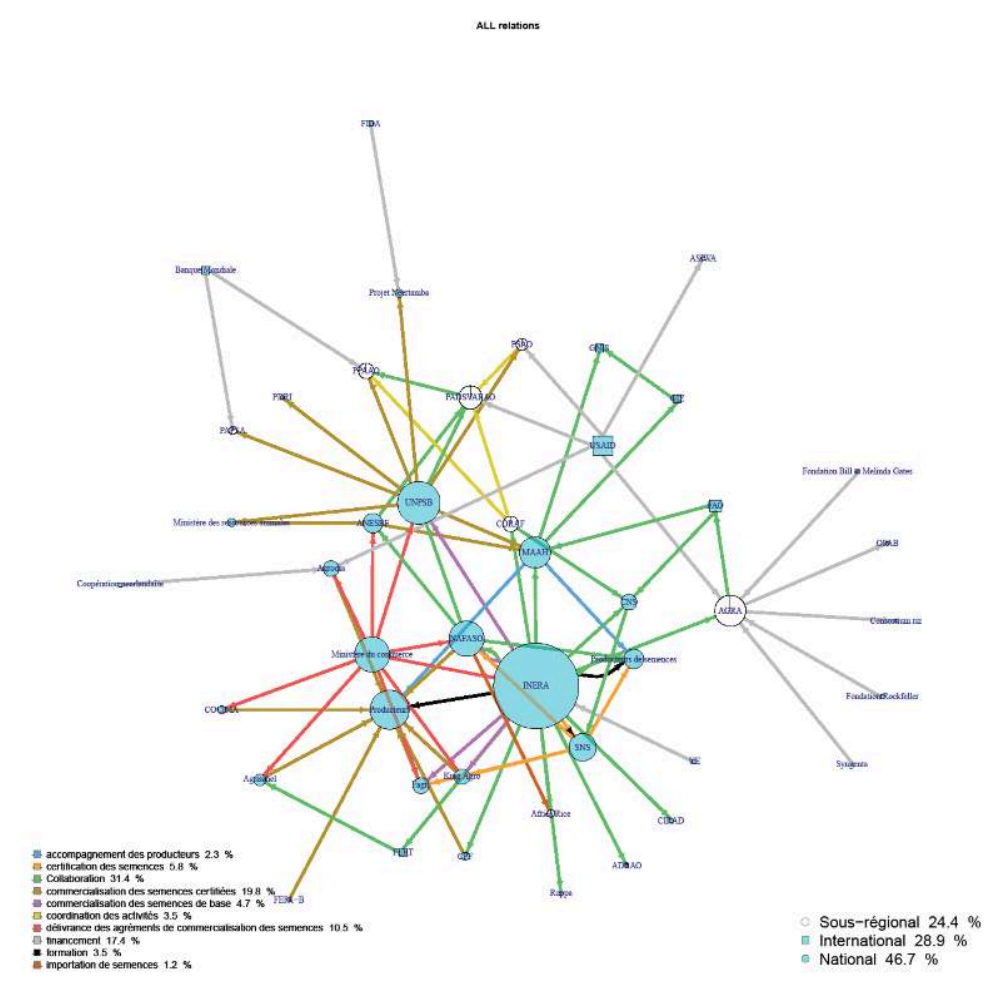

Source : Auteurs

La dernière catégorie d'acteurs est composée d'organismes de financement (Banque Mondiale, le Fonds International pour le Développement Agricole (FIDA), la FAO, les fondations Bill et Melinda Gates), qui ont peu d'interactions, mais de l'influence. Martin et Clapp (2015) ont montré que l'association historique finance-Etat-agriculture tend à se perpétuer. Les organismes de financement, au nom de l'aide et des accords bi ou multipartites, apportent leur soutien aux Etats dans la définition des orientations politiques. Ils financent des programmes, projets, alliances nationaux et sous régionaux. Les Etats à leur tour, réagissent en adaptant leurs textes, activités aux idéaux des organismes financeurs, créateurs de concepts. Cela crée un cadre favorable qui rassure les acteurs du secteur privé, et les incitent à investir. Cette situation n'est favorable que si après un certain temps, les Etats sont en mesure d'assurer eux-mêmes leur développement et d'assumer financièrement les engagements politiques qu'ils se seront fixés. Si tel n'est pas le cas, les fonds alloués dans le contexte de crise, peuvent un jour être alloués à d'autres secteurs jugés plus attractifs (Ribier et Gabas, 2016). 
Malgré la volonté dans les années 1970 de libéraliser les secteurs de l'économie, et de réduire l'impact de l'Etat sur les décisions des agents, la « financiarisation $»^{5}$ (Gabas, Ribier, et Vernières, 2017) accrue du secteur agricole ne contribue pas toujours à la mise sur pied d'initiatives de développement inclusives. Cela conduit les Etats à favoriser des cultures commerciales telles que le riz au détriment des cultures d'autoconsommation telles que le mil et le sorgho.

Tableau 4 : Part de chaque céréale dans les statistiques agricoles entre 2006 et 2013

\begin{tabular}{|l|c|c|c|c|}
\hline $\begin{array}{r}\text { Cultures } \\
\text { Indicateurs } \downarrow\end{array}$ & Riz & Maïs & Sorgho & Mil \\
\hline Part dans la production agricole totale & $2 \%$ & $6 \%$ & $11 \%$ & $8 \%$ \\
\hline Part dans la consommation totale (kg/tête/jour) & $8 \%$ & $20 \%$ & $17 \%$ & $16 \%$ \\
\hline Part dans les importations agricoles totales & $30 \%$ & $0 \%$ & $0 \%$ & $0 \%$ \\
\hline Part dans les exportations agricoles totales & $0 \%$ & $1 \%$ & $0 \%$ & $0 \%$ \\
\hline Part des dépenses spécifiques à l'agriculture & $7 \%$ & $1 \%$ & $0 \%$ & $0 \%$ \\
\hline Nombre de variétés inscrites au catalogue national & 43 & 30 & 27 & 12 \\
\hline
\end{tabular}

Source : Comité National des Semences (2014) et FAO (2014)

Le tableau 5 permet de constater que, bien qu'entre 2006 et 2013 le riz ne soit pas la céréale la plus consommée, ni même la plus produite, il occupe une plus grande part du budget spécifique à l'agriculture. Outre les documents de stratégies communs à toutes les cultures (SDR, PNSR I, et PNSR II I $^{6}$, la filière riz est encadrée par des documents qui lui sont propres à l'instar de: (i) la Stratégie nationale de développement durable de l'agriculture irriguée (SNDDAI), (ii) la première Stratégie nationale de développement de la riziculture (2011-2020), (iii) la deuxième stratégie de développement de la riziculture (élaborée en mai 2019), et (iv) le plan d'action de la filière riz.

Le riz a son propre conseil interprofessionnel (CIR-B), alors que les problématiques liées aux autres céréales et au niébé sont discutées dans le Conseil interprofessionnel des céréales (CIC-B). Un consortium riz a aussi été créé en 2017 pour faciliter la mise en relations des producteurs (représentés par leur union) avec les acheteurs. L'intérêt pour cette culture commence en 2008 avec la création de la Coalition for African Rice Development (CARD), une initiative conjointe de l'Alliance for a Green revolution in Africa (AGRA) et de la JICA, qui a financé jusqu'ici 37 projets au Burkina Faso. Parmi ces projets, on retrouve le pôle agricole de Bagré, le projet riz pluvial, le projet de développement $d u$ Sud-Ouest. Une étude réalisée en 2018 a révélé que, sur 40 producteurs enquêtés dans la zone de Bagré, tous utilisent des semences améliorées de riz, le plus souvent fournis par le pôle agricole (Ouedraogo, 2018).

51 L'intérêt croissant des bailleurs de fonds pour le riz, sous la forme d'aide au développement pousse l'Etat burkinabé à définir des politiques attractives de financement pour celui-ci, au détriment de cultures plus largement consommées. Mais selon Servet (2010, p. 10), la notion d'aide au développement est un oxymore qu'il 
convient de nuancer. Pour lui, « le développement qu'il désigne par «accumulation de capital» ne peut être possible que si l'aide n'encourage pas l'importation de biens étrangers ». Or, force est de constater que «l'aide » bilatérale liée et/ou multilatérale dans le secteur rizicole, présente tout de même une contrepartie en lien avec l'importation de riz, qui pourrait générer un cercle vicieux d'aide perpétuelle. En effet, du fait de l'instabilité politique, des conflits récurrents, et de la forte dépendance de l'agriculture aux variations climatiques, les Etats africains dont le Burkina peinent à créer la "révolution verte» observée dans d'autres pays, et donc à tirer profit véritablement de l'aide (Torretti, 2017). On aboutit à la situation inverse évoquée par Tibor (1972), dans laquelle les pays en développement paient plus qu'ils ne reçoivent (« les pauvres » financent les « riches »).

52 L'attention des organismes de financement aux semences améliorées a créé un sentier dépendant, principalement pour le riz. Mais cela n'implique pas nécessairement un verrouillage institutionnel ; ce n'est que le résultat d'une mobilisation d'acteurs qui ont su tirer parti de ce système et qui protègent leurs intérêts (Sorensen, 2015). Les autres individus continuent d'avoir un certain degré de liberté et d'autonomie dans leurs choix, et l'Etat en tant que somme d'individus maximisateurs, ne peut garantir les valeurs de justice. L'injustice étant représentée ici par l'interdiction de ventes des semences non certifiées, et le faible financement accordé aux autres céréales (mil et sorgho notamment) qui sont à la base de l'alimentation.

Conscient de cela, la société civile s'est engagée à soutenir les efforts ancestraux de préservation des semences paysannes. Ces initiatives qui ont commencé dans les années 90 , ont pris de l'ampleur depuis 2008 , à la suite d'évènements qui tendent à transformer les arrangements institutionnels existants. L'évolution progressive des évènements montre bien que les séquences réactives, caractérisées par la résistance des acteurs aux institutions dominantes, sont des chaines d'évènements ordonnés dans le temps et liées de manière causale (Mahoney, 2001).

\subsubsection{Les séquences réactives de sauvegarde des semences paysannes}

L'histoire politique du Burkina est marquée par une succession de luttes civiles pour conserver un minimum d'autonomie et d'indépendance vis-à-vis des orientations politiques des différents régimes qui se sont succédés (Loada, 1999). De la révolution burkinabé en 1983, au mouvement d'insurrection populaire en 2014, qui a conduit au départ de l'ex-président Blaise Compaoré (Chouli, 2015; Zeilig, 2017), la population burkinabé s'est toujours opposée à toutes mesures visant à réduire les libertés individuelles des citoyens. C'est dans cet ordre d'idées que naissent dans les années 90 des OPs, des associations d'encadrement d'agriculteurs familiaux, et de promotion des semences paysannes. A l'arrivée de Blaise Compaoré au pouvoir, le mouvement syndical a du mal à s'épanouir, du fait de la corruption de nombreux responsables (chefs traditionnels et médias), et de la censure médiatique (Loada, 1999). Mais l'assassinat en 1998 du journaliste et syndicaliste Norbert Zongo, l'introduction du coton BT en 2008, et la volonté de l'ex-président à changer la constitution en 2014 ont remis en avant les idéaux de justice, d'Etat de droit et de démocratie. L'année 2014 marque un tournant dans la lutte pour la préservation des semences paysannes.

Les acteurs dominants de cette lutte sont les organisations de la société civile, dont l'un des acteurs phare est le Collectif Citoyen pour l'AgroEcologie (CCAE). Né le 04 mars 2015 pendant la période de la transition, le CCAE rassemble une trentaine d'organisations 
nationales, sous régionales et internationales. A ses côtés, on retrouve des associations nationales et sous régionales telles qu'Inades formation, la Coalition pour la Protection du Patrimoine Génétique (COPAGEN), APN Sahel, le Comité Ouest Africain des Semences Paysannes (COASP), Diobass, l'Association nourrir sans détruire (ANSD) ; la liste n'étant pas exhaustive. L'ANSD a élaboré avec l'aide de l'INERA, un catalogue de semences paysannes dans l'Est du Burkina Faso.

Ces organisations interviennent dans la formation des producteurs aux pratiques agroécologiques, la création de banques de gènes communautaires, l'organisation annuelle de foires des semences paysannes, et le plaidoyer au niveau politique pour la revalorisation des semences paysannes. En 2016, elles ont rédigé avec l'aide de la Confédération Paysanne du Faso (CPF), un mémorandum pour l'insertion de l'agroécologie dans le second plan national du secteur rural (PNSR 2) (Confédération Paysanne du Faso, 2016). Leurs recommandations ont été prises en compte, et on aboutit en 2017 à la nomination d'un responsable agroécologie au ministère de l'agriculture. Leur engagement est soutenu par des partenaires extérieurs qui assurent le partage d'informations, et le relais de leurs activités au plan international. Parmi eux on compte: (i) l'association Ingalan, association de militant(e)s et acteur(rice)s breton(ne)s du commerce équitable, (ii) la via Campesina, mouvement international paysan qui rassemble 182 organisations de producteurs de 81 pays, (iii) le réseau Béninois JINUKUN, (iv) l'organisation Navdanya de Vandana Shiva, (v) le mouvement Slow food, qui défend les traditions locales.

57 La couverture médiatique de leurs activités est aussi encouragée par une gouvernance politique ouverte au dialogue avec la société civile, et par l'implication de la recherche. Des études ont déjà montré qu'en plus de leurs relations fortes avec les petits producteurs, les ONGs doivent aussi être en relation avec les services publics, la recherche et les universités pour faire entendre leurs voix (Spielman et al., 2011). Cela favorise l'émergence de dispositifs hybrides, incluant des dynamiques «top-down » et «bottom-up » (Lamine, 2012).

Les entretiens ont mis en évidence que des chercheurs de l'INERA regroupés au sein de la CONAGREP, sont impliqués depuis 2011 dans un projet de renforcement des systèmes semenciers traditionnels; la CONAGREP étant la structure chargée de la préservation des semences paysannes. Le projet est financé par la coopération Suisse, avec l'appui technique de Bioversity International, et vise à renforcer les systèmes semenciers traditionnels du mil, sorgho, et niébé ; cultures pour lesquelles le taux d'utilisation des semences certifiées reste faible en comparaison du riz et du maïs. Les chercheurs du projet reconnaissent les insuffisances de la législation nationale dans la valorisation des ressources génétiques locales performantes, à cause d'une vision focalisée sur les semences améliorées. Ils recommandent de ce fait que les initiatives de production de semences traditionnelles de qualité supérieure et de création de banques de gènes communautaires, soient aussi soutenues de la même manière que la production de semences améliorées (INERA, 2014). En 2015, ces chercheurs ont participé à la réalisation d'un documentaire sur la disparition progressive de certaines semences. Ce documentaire intitulé « Et si le tồ ${ }^{7}$ venait à disparaitre? » vise à attirer l'attention des acteurs du système semencier sur l'importance de préserver les ressources phytogénétiques. paysannes reste limité. Dans un souci d'autonomie et d'indépendance, certaines 
associations préfèrent elles-mêmes financer leurs activités dans la limite de leurs possibilités. Comme l'affirme le porte-parole du CCAE, « Le collectif tient à son autonomie et à son indépendance et se refuse à demander de l'aide à des bailleurs de fonds; les membres exercent une activité complètement bénévole ». Les quelques bailleurs de fonds identifiés durant l'étude sont la coopération Suisse, Bioversity international et le Comité catholique pour la Faim et le Développement (CCFD). En 2017, un collectif d'acteurs publics (CONAGREP) et de la société civile (COASP, la COPAGEN et APN sahel), a élaboré une loi qui a été promulguée en 2019, et qui autorise d'ores et déjà la commercialisation des semences paysannes au Burkina Faso ${ }^{8}$.

Figure 7 : Système d'innovation sur les semences paysannes

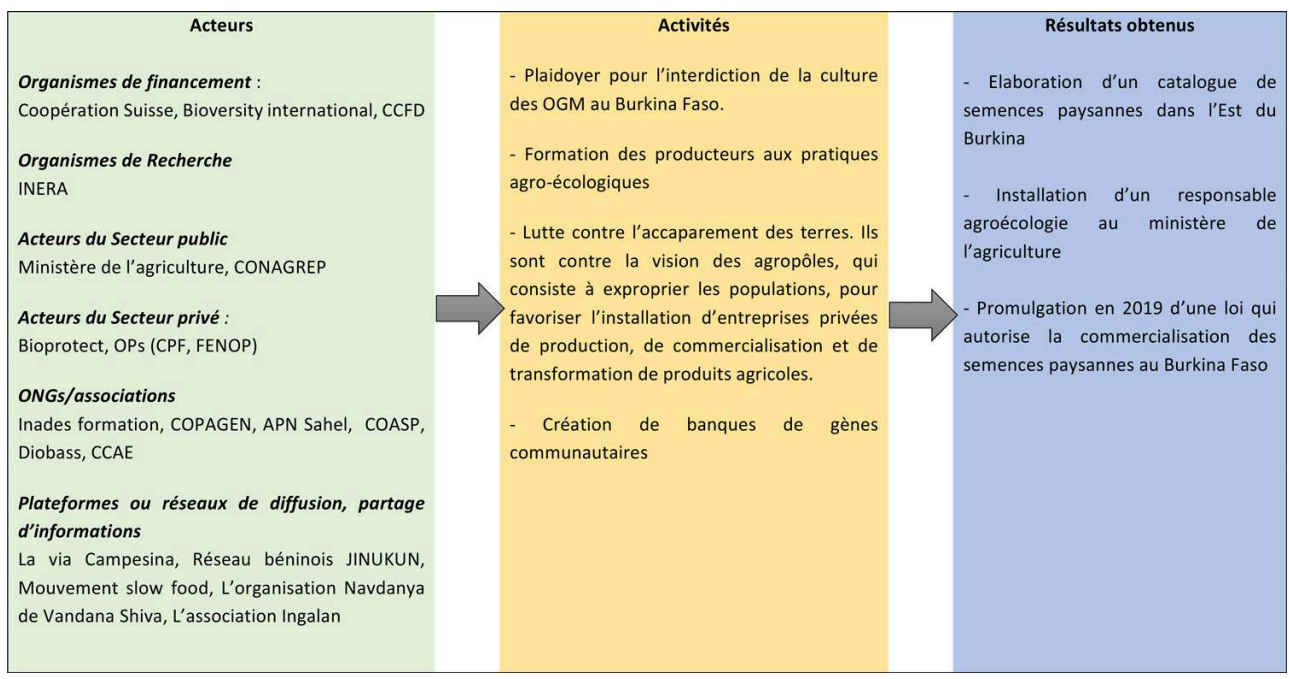

Les actions menées par la société civile favorisent l'émergence et/ou la consolidation de mécanismes d'hybridation de la gouvernance du secteur semencier. La société civile « contribue avec des acteurs locaux hétéroclites, à la diversité politique des arènes de la gouvernance, où l'Etat n'est plus aussi hégémonique qu'au lendemain des indépendances ». Des études ont mis en exergue le rôle joué par les associations dans la définition et la redéfinition d'une logique collective d'intervention, et dans l'initiation d'entreprises ou systèmes hybrides (Dufays et Huybrechts, 2016). On aboutit à une conversion institutionnelle (Mahoney et Thelen, 2010) qui se traduit non pas par l'orientation des anciennes institutions vers d'autres fins, mais par l'émergence d'une nouvelle coalition politique et une nouvelle architecture de gouvernance des trajectoires technologiques. Selon Mahoney et Thelen (2010) les acteurs à l'origine de cette coalition peuvent être considérés comme les « perdants » de l'ancien système, qui utilisent une défaillance ou une ambiguïté pour faire valoir leurs opinions.

61 A partir de 2008, co-évoluent les concepts «révolution verte » et "développement durable ", qui non loin de s'opposer, se rejoignent autour du principe de futurité, à l'origine de l'émergence d'institutions économiques. La futurité est liée à l'instinct de survie de Darwin, et émane de l'incertitude sur l'avenir. Si pour le système des semences certifiées la crainte de manquer se manifeste par des stratégies axées sur l'utilisation de variétés améliorées pour accroître la production, pour le système de semences paysannes, la crainte de détruire la biodiversité pour les générations futures a conduit au plaidoyer pour les innovations locales et à la création de banques de semences communautaires. Dans le consensus du développement, la création de 
nouveaux concepts est une arme redoutable qui s'impose presque qu'unanimement aux Etats. Si quelques-uns connaissent des échecs parce qu'inadaptés au contexte des lieux d'implantation (le cas des agropoles par exemple), d'autres en revanche permettent d'ouvrir l'arène politique à une plus grande diversité d'acteurs, à condition que les intérêts défendus soient réellement observés sur le terrain et que ces mots ne soient pas des mots parapluies pour canaliser ou contrôler une population.

\section{Conclusion}

L'objectif de cet article était de tester l'hypothèse d'une dépendance de sentier du système des semences certifiées au Burkina Faso, qui aurait abouti à un verrouillage institutionnel de systèmes alternatifs. L'analyse par triangulation des données issues des entretiens, de la revue documentaire, et du forum conduit à des résultats qui infirment cette hypothèse, dans la mesure où nous n'observons pas de verrouillage. La mobilisation autour des semences certifiées se situe en phase 2 (formation du sentier) de la constitution du sentier, et est axée majoritairement sur les productions de riz et de maïs. Cette gouvernance est pilotée par une interdépendance des acteurs et des cadres nationaux, régionaux et internationaux, qui favorise l'accumulation d'actifs et le développement $d u$ secteur privé. Les acteurs ont graduellement mis en place des stratégies pour préserver les avantages (économiques et politiques) tirés de ce secteur, et renforcer sa trajectoire. La stricte distinction entre «système formel » et «système informel » fait partie intégrante de ces stratégies, puisqu'elle est instrumentée pour distinguer les « bonnes » des « mauvaises " pratiques.

Cette concentration comporte des risques en termes de développement dans la mesure où elle homogénéise les institutions, les dynamiques entrepreneuriales, qui gouvernent les sources de la biodiversité cultivée. La biodiversité étant au cœur des capacités d'innovation pour faire face aux instabilités du futur, cette trajectoire est remise en question au Burkina Faso, dans un contexte de démocratisation effective de la vie publique. L'on a progressivement abouti à une conception du changement et du développement qui ne tient pas compte uniquement de la maximisation monétaire du rapport coûts/avantages, mais plutôt des préférences sociales collectives. Par le biais de plaidoyer/médiation pour les innovations locales, la société civile modifie les mécanismes de gouvernance du système semencier en incorporant de nouveaux acteurs dans la sphère politique.

L'exemple du Burkina Faso met en lumière la coexistence des mots du développement pour orienter une trajectoire. Alors que dans les années 60 le développement rimait avec "croissance économique», dans les années 90 le concept de «bonne gouvernance » incite les acteurs à réduire les dépenses publiques dans l'agriculture. En 2008, les idéaux de croissance économique sont remis à l'ordre de jour, sous le vocable de «révolution verte». Malgré les effets climatiques de ce dernier dans les pays européens, il est soutenu avec l'argument que, s'il est contrôlé et mieux encadré que dans le passé, les conséquences ne peuvent être aussi dramatiques. Pendant ce temps, la sélection massale et le troc continuent d'exister, de se perpétuer, évoluant dans une logique qui ne tient compte que des individus qu'ils desservent, et peu soucieuses des attentes et volontés des organisations internationales, créatrices de concepts. La célébration de l'année de l'agriculture familiale en 2014 institutionnalise la reconnaissance de l'agroécologie. Pourtant déjà appliquée par des populations rurales 
depuis des millénaires, il se généralise comme le moyen de réduire les effets de l'intensification agricole sur l'environnement, la biodiversité et la santé des consommateurs. A ce concept est ajouté, celui d'inclusion sociale, pour rappeler l'importance du « gouvernement par le bas ». Les concepts « d'intensification agricole » et "d'agroécologie » connaissent ainsi une évolution conjointe, tant au niveau des instances internationales, qu'au niveau des Etats. Dans le secteur semencier céréalier au Burkina Faso, cela favorise la coexistence de deux institutions, le marché pour le riz et le maïs, et l'échange non marchand pour le mil et le sorgho. Cela contribue à enrichir la gouvernance $\mathrm{du}$ système semencier, en $\mathrm{y}$ incorporant de nouvelles formes d'institutions et d'organisations.

\section{BIBLIOGRAPHIE}

African Centre for biodiversity. (2015). The expansion of the commercial sector in sub-saharan Africa : Major players, key issues and trends.

Allal, A. (2010). « Quand l'essentiel est de participer ». Sociologie politique de projets internationaux de développement au Maroc et en Tunisie. Économie et institutions, 14, 95-116. https://doi.org/10.4000/ei.199

Almekinders, C. (2000). The importance of informal seed sector and its relation with the legislative framework. 16.

Almekinders, C. J., Louwaars, N. P., \& De Bruijn, G. H. (1994). Local seed systems and their importance for an improved seed supply in developing countries. Euphytica, 78(3), 207-216.

Ansaloni, M. (2017). Le marché comme instrument politique. Societes contemporaines, $\mathrm{N}^{\circ} 105(1)$, 79-102.

Beckmann, V., \& Padmanabhan, M. (2009). Analysing Institutions : What Method to Apply? In V. Beckmann \& M. Padmanabhan (Éds.), Institutions and Sustainability (p. 341-371). Springer Netherlands. https://doi.org/10.1007/978-1-4020-9690-7_16

Bennett, A., \& Elman, C. (2006). Complex Causal Relations and Case Study Methods : The Example of Path Dependence. Political Analysis, 14(03), 250-267. https://doi.org/10.1093/pan/mpj020

Buton, F., Lehingue, P., Mariot, N., \& Rozier, S. (2016). L' Ordinaire du Politique : Enquêtes sur les rapports profanes au politique. Presses Univ. Septentrion.

Callon, M., \& Barthe, Y. (2005). Décider sans trancher. Negociations, no 4(2), 115-129.

Casadella, V., \& Tahi, S. (2017). Capacités et politiques d'innovation dans les pays moins avancés : Enseignements tirés du cas du Sénégal. Innovations, n 53(2), 13-39.

CEDEAO. (2017). Evaluation de la mise en oeuvre du règlement semencier régional.

CEDEAO, CILSS, \& UEMOA. (2015). Plan d'action Comité Régional des Semences (COASEM et CRSU) (Programme semencier pour l'Afrique de l'Ouest).

Chouli, L. (2015). L'insurrection populaire et la Transition au Burkina Faso. Review of African Political Economy, 42(143), 148-155. https://doi.org/10.1080/03056244.2015.1016290 
Christinck, A., Diarra, M., \& Horneber, G. (2014). Innovation dans les systèmes semenciers. Leçons tirées du projet « appui aux initiatives semencières gérées par les paysans au Mali, au Niger et au Burkina Faso ». Fondation McKnight. http://www.ccrp.org/sites/default/files/ ccrp_seedsystems_french.pdf

Comité National des Semences. (2014). Catalogue national des espèces et variétés agricoles au Burkina Faso.

Confédération Paysanne du Faso. (2016). Mémorandum des organisations professionnelles agricoles du Burkina Faso. Note de contribution à l'élaboration du programme national du secteur rural (PNSR) phase 2.

CORAF, CEDEAO, \& USAID. (2014). Contribution to the CAADP Process Regional Seed Policy and Famer Access to Quality Seeds in West Africa (p. 11). CORAF. http://www.coraf.org/wasp2013/ wp-content/uploads/2013/07/SEED_POLICY_REFORMS_AND_SEED_USE.pdf

Coulibaly, M., \& Brac de la Perrière, R. A. (2019). Faillite de la protection intellectuelle des obtentions végétales : 10 années d'UPOV en Afrique francophone.

David, P. A. (1994). Why are institutions the 'carriers of history'? : Path dependence and the evolution of conventions, organizations and institutions. Structural Change and Economic Dynamics, 5(2), 205-220. https://doi.org/10.1016/0954-349X(94)90002-7

David, P. A. (2007). Path dependence : A foundational concept for historical social science. Cliometrica, 1(2), 91-114. https://doi.org/10.1007/s11698-006-0005-x

Demeulenaere, E. (2013). Les semences entre critique et expérience : Les ressorts pratique d'une contestation paysanne. Review of Agricultural and Environmental Studies, 94(4), 421-442.

Djamen, P. (2016). Developing the seed industry to increase agricultural productivity in West and Central Africa. Keys intervention and guiding principles [Policy brief].

Dobusch, L., \& Kapeller, J. (2013). Breaking New Paths : Theory and Method in Path Dependence Research. Schmalenbach Business Review, 65(3), 288-311. https://doi.org/10.1007/BF03396859

Dosi, G. (1982). Technological paradigms and technological trajectories : A suggested interpretation of the determinants and directions of technical change. Research Policy, 11(3), 147-162. https://doi.org/10.1016/0048-7333(82)90016-6

Dufays, F., \& Huybrechts, B. (2016). Where do hybrids come from? Entrepreneurial team heterogeneity as an avenue for the emergence of hybrid organizations. International Small Business Journal, 34(6), 777-796. https://doi.org/10.1177/0266242615585152

FAO. (2014a). Analyse des dépenses publiques en soutien à l'agriculture et au développement rural au Burkina Faso (Programme de Suivi et Analyses des Politiques Agricoles et Alimentaire (SAPAA), p. 26-53) [Série de notes techniques]. FAO. http://www.fao.org/3/a-i4513f.pdf

FAO. (2014b). Synthèse des réalisations des projets GCP/052/BKF/EC et OSRO/BKF/101/EC financés par l'Union Européenne et mis en oeuvre par la FAO. Food and Agriculture Organization.

Frydman, R. (1988). La Théorie Générale de Keynes : Economie et Politique. Cahiers d'Économie Politique, 14(1), 99-109. https://doi.org/10.3406/cep.1988.1061

Gabas, J.-J., Ribier, V., \& Vernières, M. (2017). Présentation. Financement ou financiarisation du développement? Une question en débat. Mondes en developpement, $n^{\circ}$ 178(2), 7-22.

Garud, R., Kumaraswamy, A., \& Karnøe, P. (2010). Path Dependence or Path Creation? Journal of Management Studies, 47(4), 760-774. https://doi.org/10.1111/j.1467-6486.2009.00914.X 
Gerring, J. (2004). What Is a Case Study and What Is It Good for? American Political Science Review, 98(02), 341-354. https://doi.org/10.1017/S0003055404001182

GFA Consulting Group. (2016). Etude sur les semences, rapport du Burkina Faso. GIZ.

Gill, T. B., Bates, R., Bicksler, A., Burnette, R., Ricciardi, V., \& Yoder, L. (2013). Strengthening Informal Seed Systems To Enhance Food Security in Southeast Asia. Journal of Agriculture, Food Systems, and Community Development, 3(3), 139-153-139-153. https://doi.org/10.5304/jafscd. 2013.033.005

Gislain, J.-J. (2017). Futurité, origine des institutions économiques. Économie et institutions, 25. https://doi.org/10.4000/ei.5828

Greenwood, R., Suddaby, R., \& Hinings, C. R. (2002). Theorizing Change : The Role of Professional Associations in the Transformation of Institutionalized Fields. The Academy of Management Journal, 45(1), 58-80. https://doi.org/10.2307/3069285

Groupe Crucible II, \& Foundation, D. H. (2001). Le débat des semences. IDRC.

Hayek, F. A. (2014). The Constitution of Liberty. Routledge. https://doi.org/ $10.4324 / 9781315832081$

Holtzman, J. S., Kaboré, D., Tassembedo, M., \& Adomayakpor, R. (2013). Burkina Faso : Indicateurs de l'agro-busisness [Rapport d'étude]. Banque Mondiale.

Hugon, P. (2014). L'« informel » ou la petite production marchande revisités quarante ans après. Mondes en developpement, $\mathrm{n}^{\circ}$ 166(2), 17-30.

Ilbert, H., \& Louafi, S. (2004). Biodiversité et ressources génétiques : La difficulté de la constitution d'un régime international hybride. Tiers-Monde, 45(177), 107-128. https://doi.org/ 10.3406/tiers.2004.5450

INERA. (1998). Bilan de 10 années de recherche. Institut National de l'Environnement et la Recherche Agricole.

INERA. (2014). Rapport national consolidé du projet « renforcement des systèmes semenciers traditionnels pour réduire les risques d'échec des cultures au sahel » (p. 68-78). Institut National de l'Environnement et la Recherche Agricole, Bioversity International.

James, É. (1955). Histoire de la pensée économique au XXe siècle (1) : De 1900 à la théorie générale de J. M. Keynes 1936. FeniXX.

Kaboré, R., Dabat, M.-H., \& Vom Brocke, K. (2008). Innovation technique et organisation des acteurs : Le cas des semences certifiées dans le Sanmatenga au Burkina Faso, retour sur une expérience d'une dizaine années (1998-2008). 8. http://agritrop.cirad.fr/557969/1/ document_557969.pdf

Koussoubé, E., Loada, A., Nebié, G., \& Raffinot, M. (2015). Economie politique de la croissance au Burkina Faso : Institutions, gouvernance et développement. (p. 10-12) [Document de travail]. Univesité Paris Dauphine, IRD.

Kpadé, P. C., \& Boinon, J.-P. (2011). Dynamique des politiques cotonnières au Bénin. Une lecture par la dépendance de sentier. Économie rurale. Agricultures, alimentations, territoires, 321, 58-72. https://doi.org/10.4000/economierurale.2949

Krief, N., \& Zardet, V. (2013). Analyse de données qualitatives et recherche-intervention. Recherches en Sciences de Gestion, $N^{\circ}$ 95(2), 211-237. 
Lamine, C. (2012). «Changer de système » : Une analyse des transitions vers l'agriculture biologique à l'échelle des systèmes agri-alimentaires territoriaux. Terrains travaux, $n^{\circ} 20(1)$, 139-156.

Laperche, B. (2009). Stratégies d'innovation des firmes des sciences de la vie et appropriation des ressources végétales : Processus et enjeux. Mondes en développement, 3(147), 109-122. https:// doi.org/10.3917/med.147.0109

Laperche, B. (2018). Le capital savoir de l'entreprise. ISTE Group.

Lazaric, N. (2010). Les théories économiques évolutionnistes (La découverte). https:// www.cairn.info/les-theories-economiques-evolutionnistes--9782707147202.htm

Loada, A. (1999). Réflexions sur la société civile en Afrique : Le Burkina de l'après-Zongo. Politique africaine, $\mathrm{N}^{\circ} 76(4), 136-151$.

Louwaars, N. (2007). Seeds of confusion : The impact of policies on seed systems.

Louwaars, N., Le Coent, P., \& Osborn, T. (2011). Seed systems and plant genetic resources to food and agriculture. Food and Agriculture Organization of The United Nations. http://www.fao.org/ fileadmin/templates/agphome/documents/PGR/SoW2/tbs_Seed_Systems_081209.pdf

MAAH, GIZ, \& GNIS. (2018). IProjet d'accord de coopération tripartite entre le Ministère de l'Agriculture et des Aménagements Hydrauliques du Burkina Faso (MAAH), le Projet Centre d'Innovations Vertes (ProCIV) de la Deutsche Gesellschaftfür Internationale Zusammenarbeit (GIZ) et le Groupement National Interprofessionnel des Semences et plants (Gnis) de la France pour appuyer l'adhésion du Burkina Faso aux systèmes des semences de l'OCDE, de l'Union Européenne, et à l'accréditation ISTA.

Maggetti, M. (2014). Le changement institutionnel et l'évolution de l'État régulateur : L'exemple suisse. Revue Internationale des Sciences Administratives, Vol. 80(2), 281-303.

Magnani, N., \& Osti, G. (2016). Does civil society matter? Challenges and strategies of grassroots initiatives in Italy's energy transition. Energy Research \& Social Science, 13, 148-157. https:// doi.org/10.1016/j.erss.2015.12.012

Magrin, G. (2015). L’Afrique entre « malédiction des ressources » et « émergence » : Une bifurcation? Revue Francaise de Socio-Economie, Hors-série(2), 105-120.

Mahoney, J. (2001). Path-Dependent Explanations of Regime Change : Central America in Comparative Perspective. Studies in Comparative International Development, 36(1), 111-141. https://doi.org/10.1007/BF02687587

Mahoney, J., \& Thelen, K. (2010). A Gradual Theory of Institutional Change. Explaining Institutional Change: Ambiguity, Agency, and Power. https://www.scholars.northwestern.edu/ en/publications/a-gradual-theory-of-institutional-change

Manzelli, M., \& Laghetti, G. (2014). Le secteur semencier dans la moyenne Casamance : Aperçu sur l'organisation et les limitations de la filière des semences de riz (p. 8-15). http:// www.papsen.org/data/files/PAPSEN_9_SEMENCES_0214.pdf

Marques Pereira, J., \& Ould-Ahmed, P. (2010). Le pouvoir des mots dans le nouveau consensus du développement. Un nouveau mode de gouvernement des pauvres. Économie et institutions, 14, 5-14. https://doi.org/10.4000/ei.230

Martin, S. J., \& Clapp, J. (2015). Finance for Agriculture or Agriculture for Finance? Journal of Agrarian Change, 15(4), 549-559. https://doi.org/10.1111/joac.12110 
MASA, \& FAO. (2014). Rapport final de l'étude portant sur la détermination des indicateurs des semences de variétés améliorées au Burkina Faso (p. 10) [Rapport d'étude]. Ministère de l'Agriculture et de la Sécurité Alimentaire.

McGuire, S., \& Sperling, L. (2016). Seed systems smallholder farmers use. Food Security, 8(1), 179-195. https://doi.org/10.1007/s12571-015-0528-8

Ministère de l'Agriculture, de l'Hydraulique et des Ressources Halieutiques. (2011). Diagnostic du secteur semencier au Burkina Faso.

Ministère de L'agriculture et de la Sécurité Alimentaire. (2013). Etude de faisabilité pour la création d'une société de production de semences de riz au Burkina Faso.

Ministère de l'agriculture et des aménagements hydrauliques. (2010). Stratégie de développement durable du secteur semencier du Burkina Faso 2011-2020.

Ministère de l'agriculture et des ressources animales, \& FAO. (1992). Politique et programme de développement du secteur semencier. Ministère de l'agriculture et des ressources animales.

Moedas, C. (2017). La biotechnologie industrielle, facteur de développement durable et de croissance verte. Annales des Mines-Réalités industrielles, 5-7.

Muok, B. O., \& Kingiri, A. (2015). The role of civil society organizations in low-carbon innovation in Kenya. Innovation and Development, 5(2), 207-223. https://doi.org/10.1080/2157930X. 2015.1064558

Ndoye, A. (2018, mars 27). Burkina Faso : Une banque agricole en route. Financial Afrik. https:// www.financialafrik.com/2018/03/27/burkina-faso-une-banque-agricole-en-route/

Nelson, R. R. (2009). An Evolutionary Theory of Economic Change. Harvard University Press.

North, D. C. (1990). Institutions, Institutional Change and Economic Performance. Cambridge University Press.

North, D. C. (1995). The New Institutional Economics and Third World Development. In The New Institutional Economics and Third World Development (John Harriss, Janet Hunter, Colin Lewis, p. 17-26). Routledge. https://doi.org/10.4324/9780203444290-7

Nubukpo, K. (2013). Quel futur pour l'Afrique ? Evolution des paradigmes du développement, débats méthodologiques et perspectives. L’Economie politique, n 59(3), 76-90.

Ouedraogo, T. L. (2011). L'agro business et la sécurisation foncière entre mutations agricoles et dynamiques foncières dans la commune rurale de Cassou (Burkina Faso) [Mémoire de master 2 recherche en géographie]. Université Paris 1 Panthéon Sorbonne.

Pesqueux, Y. (2012). L'économie informelle, une bonne « mauvaise pratique » ? Revue francaise de gestion, $\mathrm{N}^{\circ} 228-229(9), 217-229$.

Philip, P. (1995). Transcendental realism - a foundation for evolutionary economics? International Journal of Social Economics, 22(12), 19-35. https://doi.org/ 10.1108/03068299510104723

Pierson, P. (2000). Increasing Returns, Path Dependence, and the Study of Politics. American Political Science Review, 94(2), 251-267. https://doi.org/10.2307/2586011

Polanyi, K., \& MacIver, R. M. (1944). The great transformation (Beacon Press, Vol. 2).

République du Burkina Faso. (2019). Décret n²019-0619/PRES promulguant la loi n020-2019/AN du 07 mai 2019 portant accès aux ressources phytogénétiques pour l'alimentation et l'agriculture et au partage des avantages résultant de leur utilisation, p. 2. 
République du Burkina Faso. (2017). Deuxième Programme National du Secteur Rural (PNSR) 2016-2020.

République du Burkina Faso. (2012). Programme National du Secteur Rural (PNSR) 2011-2020.

République du Burkina Faso. (2009). Décret n²009-403/PRES/PM/MESSRS/MECV/MAHRH/MRA/ MEF du 10 juin 2009 portant création, attributions, organisation et fonctionnement d'une commission nationale de gestion des ressources phytogénétiques (CONAGREP), Pub. L. No. 2009-403, p. 2 http://www.fao.org/faolex/results/details/en/c/LEX-FAOC092254/

République du Burkina Faso. (2006). Loi n 010-2006/AN Portant Reglementation des semences végétales au Burkina Faso, Pub. L. No. 010-2006/AN, p. 16.

Réseau mondial pour le droit à l'alimentation et à la nutrition, \& Convergence globale des luttes pour la terre et l'eau. (2018). Bénéfices des entreprises ou diversité des systèmes alimentaires? Les menaces pesant sur les semences paysannes et leurs implications en Afrique de l'Ouest. FIAN International. https://www.fian.org/fileadmin/user_upload/ 180208_FIAN_FFM_BurkinaFaso_Web.pdf

Ribier, V., \& Gabas, J.-J. (2016). De nouveaux instruments financiers pour le développement agricole en Afrique de l'Ouest? Techniques Financieres et Developpement, $n^{\circ}$ 124(3), 53-65.

Sawadogo M.F.W. E., Temple L. , Ouedraogo S., Zongo A, Soulama S. (2020). Renouveler la gouvernance du secteur semencier au Burkina Faso pour un développement inclusif. Policy Brief $\mathrm{n}^{\circ} 1, \mathrm{dP}$ Information pour la sécurité alimentaire, Projet Coex - Cirad Montpellier, $4 \mathrm{p}$.

Sanfo, S. (2010). Politiques publiques agricoles et lutte contre la pauvreté au Burkina Faso : Le cas de la région du Plateau central [Thesis, Paris 1]. http://www.theses.fr/2010PA010023

Semaan, S. (2010). Échantillonnage espace-temps et échantillonnage déterminé selon les répondants des populations difficiles à joindre. Methodological Innovations Online, 5(2), 60-75. https://doi.org/10.4256/mio.2010.0019a

Serageldin, I. (1999). Biotechnology and Food Security in the 21st Century. Science, 285(5426), 387-389. https://doi.org/10.1126/science.285.5426.387

Serageldin, I. (2011). Mobiliser le savoir pour éradiquer la faim. Collège de France.

Servet, J.-M. (2010). Aide au développement : Six décennies de trop dits et de non dits. Revue de la régulation. Capitalisme, institutions, pouvoirs, 7. https://doi.org/10.4000/regulation.7813

Sorensen, A. (2015). Taking path dependence seriously : An historical institutionalist research agenda in planning history. Planning Perspectives, 30(1), 17-38. https://doi.org/ 10.1080/02665433.2013.874299

SPAAA, \& FAO. (2013). Revue des politiques agricoles et alimentaires au Burkina Faso.

Sperling, L., \& McGuire, S. (2010). Understanding and strengthening informal seed markets. Experimental Agriculture, 46(02), 119-136.

Spielman, D. J., Davis, K., Negash, M., \& Ayele, G. (2011). Rural innovation systems and networks : Findings from a study of Ethiopian smallholders. Agriculture and Human Values, 28(2), 195-212. https://doi.org/10.1007/s10460-010-9273-y

Sydow, J., Schreyögg, G., \& Koch, J. (2005). Organizational Paths : Path Dependency and Beyond. Sydow, J., Windeler, A., Müller-Seitz, G., \& Lange, K. (2012). Path Constitution Analysis : A Methodology for Understanding Path Dependence and Path Creation. Business Research, 5(2), 155-176. https://doi.org/10.1007/BF03342736 
Temple, L., Chiffoleau, Y., \& Touzard, J.-M. (2018). Une histoire de l'innovation et de ses usages dans l'agriculture (Editions Quae, p. 19-37). Faure Guy, Chiffoleau Yuna, Goulet Frédéric, Temple Ludovic, Touzard Jean-Marc.

Temple, L., Ndzesop, N. M., Fouepe, G. H. F., Nkeng, M. N., \& Mathé, S. (2017). Système National de Recherche et d'Innovation en Afrique : Le cas du Cameroun. Innovations, $n^{\circ}$ 53(2), 41-67.

The African Trade investment program. (2002). Une évaluation des secteurs engrais et des semences au Burkina Faso.

Thelen, K. (2003). Comment les institutions évoluent: Perspectives de l'analyse comparative historique. Presses de Sciences Po. https://www.cairn.info/l-annee-de-la-regulationn-7-2003-2004--9782724609255-page-13.htm

Tibor, M. (1972). De l'aide à la recolonisation. Les leçons d'un échec in-8º br. Seuil.

Torretti, C. (2017). Introduction à une géopolitique du riz. Les Cahiers d'Outre-Mer. Revue de géographie de Bordeaux, LXX(275), 5-17.

Zeilig, L. (2017). Burkina Faso : From Thomas Sankara to popular resistance. Review of African Political Economy, 44(151), 155-164. https://doi.org/10.1080/03056244.2016.1251200

Zheng, W., Singh, K., \& Mitchell, W. (2015). Buffering and enabling : The impact of interlocking political ties on firm survival and sales growth. Strategic Management Journal, 36(11), 1615-1636. https://doi.org/10.1002/smj.2301

\section{ANNEXES}

\section{Annexe 1 : Liste complète des organisations interviewées}

\begin{tabular}{|c|c|c|c|}
\hline Sous-systèmes & $\begin{array}{l}\text { Catégories } \\
\text { d'organisations }\end{array}$ & Organisations & $\begin{array}{l}\text { Nombre } \\
\text { d'entretiens }\end{array}$ \\
\hline \multirow{3}{*}{$\begin{array}{l}\text { Sous - système } \\
\text { recherche }\end{array}$} & \multirow[b]{2}{*}{ Universités } & Université de Ouagadougou & 1 \\
\hline & & $\begin{array}{l}\text { Institut Supérieur des Sciences et } \\
\text { technologies Agricoles (ISSTA) }\end{array}$ & 1 \\
\hline & Centre de recherche & $\begin{array}{l}\text { Institut de l'Environnement et de } \\
\text { Recherches Agricoles (INERA) }\end{array}$ & 5 \\
\hline \multirow[t]{3}{*}{$\begin{array}{l}\text { Sous - système } \\
\text { intermédiation }\end{array}$} & \multirow[t]{3}{*}{$\begin{array}{l}\text { Ministère et structures de } \\
\text { régulation }\end{array}$} & $\begin{array}{l}\text { Ministère de l'agriculture: } \\
\text { Service National des Semences } \\
\text { (SNS) }\end{array}$ & 2 \\
\hline & & $\begin{array}{l}\text { Ministère de l'agriculture : } \\
\text { Responsable agroécologie }\end{array}$ & 1 \\
\hline & & $\begin{array}{l}\text { Ministère de l'agriculture : } \\
\text { Direction des intrants }\end{array}$ & 1 \\
\hline
\end{tabular}




\begin{tabular}{|c|c|c|}
\hline & $\begin{array}{l}\text { Comité National des Semences } \\
(\mathrm{CNS})\end{array}$ & 1 \\
\hline & $\begin{array}{l}\text { Agence National de Biosécurité } \\
\text { (ANB) }\end{array}$ & 1 \\
\hline Projet & Projet riz pluvial & 1 \\
\hline & $\begin{array}{l}\text { Conseil Interprofessionnel des } \\
\text { Céréales du Burkina Faso }\end{array}$ & 1 \\
\hline Interprofessions & $\begin{array}{l}\text { Conseil Interprofessionnel du Riz } \\
\text { du Burkina Faso (CIRB) }\end{array}$ & 1 \\
\hline & Consortium Riz & 1 \\
\hline \multirow{11}{*}{ ONGs et associations } & $\begin{array}{l}\text { APN Sahel (Association pour la } \\
\text { protection de la Nature) }\end{array}$ & 1 \\
\hline & Inades formation & 1 \\
\hline & Diobass & 1 \\
\hline & Fert & 1 \\
\hline & $\begin{array}{l}\text { Secrétariat permanent des ONG } \\
(\text { SPONG) }\end{array}$ & 1 \\
\hline & $\begin{array}{l}\text { Association Nourrir Sans } \\
\text { Détruire (ANSD) }\end{array}$ & 1 \\
\hline & Autre terre & 1 \\
\hline & Ensemble Sauvons le Futur (ESF) & 1 \\
\hline & FIAN & 1 \\
\hline & $\begin{array}{l}\text { Collectif Citoyen pour } \\
\text { l'AgroEcologie (CCAE) }\end{array}$ & 1 \\
\hline & $\begin{array}{l}\text { Conseil National de l'Agriculture } \\
\text { Biologique (CNABIO) }\end{array}$ & 1 \\
\hline \multirow{3}{*}{ OPs } & $\begin{array}{l}\text { Réseau des Organisations } \\
\text { Paysannes et de Producteurs } \\
\text { d'Afrique de l'Ouest (ROPPA) }\end{array}$ & 1 \\
\hline & $\begin{array}{l}\text { Fédération Nationale des } \\
\text { Organisations de Producteurs } \\
\text { (FENOP) }\end{array}$ & 1 \\
\hline & $\begin{array}{l}\text { Confédération Paysanne du Faso } \\
\text { (CPF) }\end{array}$ & 1 \\
\hline
\end{tabular}




\begin{tabular}{|c|c|c|c|}
\hline \multirow{5}{*}{$\begin{array}{l}\text { Sous - système } \\
\text { entrepreneurial }\end{array}$} & \multirow{4}{*}{$\begin{array}{l}\text { Entreprises, unions et } \\
\text { associations de production } \\
\text { de semences }\end{array}$} & $\begin{array}{l}\text { Union Nationale des Producteurs } \\
\text { de Semences du Burkina Faso } \\
\text { (UNPSB) }\end{array}$ & 1 \\
\hline & & $\begin{array}{l}\text { Association Nationale des } \\
\text { Entreprises Semencières du } \\
\text { Burkina Faso (ANESBF) }\end{array}$ & 1 \\
\hline & & $\begin{array}{l}\text { Neema Agricole du Faso } \\
\text { (NAFASO) }\end{array}$ & 1 \\
\hline & & Bioprotect & 1 \\
\hline & Fournisseurs d'intrants & $\begin{array}{l}\text { Association des Grossistes et } \\
\text { Détaillants d'Intrants Agricoles } \\
\text { (AGRODIA) }\end{array}$ & 1 \\
\hline Bailleurs de fonds & & $\begin{array}{l}\text { Food and Agriculture Organization } \\
\text { (FAO) }\end{array}$ & 1 \\
\hline
\end{tabular}

\section{NOTES}

1. Festival alimenterre ( https://www.alimenterre.org/burkinabe-bounty-agroecologie-auburkina-faso)

Slow food (https://www.slowfood.com/fr/burkinabe-bounty-la-resistance-des-communautespour-la-souverainete-alimentaire/)

2. La démarche constructiviste se caractérise par une forte interaction du chercheur avec les acteurs du terrain (Krief \& Zardet, 2013)

3. D'après un classement fait par la CEDEAO en 2017 (CEDEAO, 2017)

4. ISTA standard https://www.seedtest.org/upload/cms/user/

ISTAAccreditationStandardforSeedTestingandSeedSamplingV6.11.pdf

ISTA passport http://issues.ink/ista/accreditation_programme/files/index.html

5. «La financiarisation du développement agricole se traduit par la multiplication des outils de financement combinant des fonds publics et des fonds privés, tels que les fonds de garantie et les fonds d'investissement " (Gabas, Ribier, et Vernières, 2017, p.18)

6. SDR (stratégie de développement du secteur rural), PNSR I (premier programme national du secteur rural), PNSR II (second programme national du secteur rural)

7. Plat national burkinabé fait traditionnellement à base de farine de mil ou de sorgho

8. (Décret $n^{\circ} 2019-0619 /$ PRES promulgant la loi n020-2019/AN du 07 mai 2019 portant accès aux ressources phytogénétiques pour l'alimentation et l'agriculture et au partage des avantages résultant de leur utilisation, 2019). 


\section{RÉSUMÉS}

La régulation semencière centrée sur des semences distinctes, homogènes et stables dans de nombreux pays industriels pousse à s'interroger sur le devenir des semences paysannes, largement répandues en Afrique. Ce phénomène est analysé au Burkina Faso sous l'angle de la dépendance de sentier. Le cadre méthodologique est structuré autour de 37 entretiens semidirectifs, d'une analyse documentaire, et de l'organisation d'un forum multi-acteurs avec 33 participants. Les résultats mettent en évidence une persistance structurelle du système des semences certifiées pour certaines céréales (maïs et riz), qui est due à une forte mobilisation des organismes de financement, l'accumulation d'actifs, et la consolidation d'un secteur privé d'approvisionnement en intrants. Toutefois, ces initiatives n'ont pas réussi à créer de verrouillage institutionnel, ce dont rend compte l'émergence de mouvements de préservation des semences paysannes. Portés par la société civile, ces mouvements contribuent à enrichir le débat des semences, en créant des plateformes et des lieux de discussion (foire des semences paysannes) sur le sujet, et de nouveaux produits (catalogue de semences paysannes). Du déplacement institutionnel matérialisé par l'importation de standards étrangers pour réguler le secteur semencier, le régime sociotechnique s'oriente vers une conversion institutionnelle caractérisée par l'émergence d'un nouvel ordre politique qui hybride différentes formes d'institutions et d'organisations.

Seed regulation based on distinct, homogenous and stable seeds in many industrial countries raises questions about the future of farmers' seeds, widely used in Africa. We analyze this phenomenon in Burkina Faso from the perspective of path dependency. The methodological framework is structured around 37 semi-structured interviews, a literature review, and the organization of a multi-stakeholder forum with 33 participants. Results highlight a structural persistence of the certified seed system for some cereals (maize and rice) due to a strong mobilization of funding agencies, an accumulation of assets, and a consolidation of a private input supply sector. However, these initiatives have not led to institutional lock-in, reflecting the emergence of farmers' seed preservation movements. Driven by civil society, these movements enrich the seed debate by creating platforms and areas for discussion (farmers' seed fair) on the subject and new products (farmers' seed catalogue). From the institutional shift materialized by the importation of foreign standards to regulate the seed sector, the socio-technical regime is moving towards an institutional conversion characterized by the emergence of a new political order that hybridizes different forms of institutions and organizations.

\section{INDEX}

Mots-clés : système semencier, innovation variétale, verrouillage technologique, Burkina Faso

Keywords : seed system, varietal innovation, technological lock-in, Burkina Faso

\section{AUTEURS}

\section{ANNY LUCRÈCE NLEND NKOTT}

Innovation, Université de Montpellier, CIRAD, INRAE, Montpellier SupAgro, Montpellier, France CIRAD, UMR Innovation, F-34398 Montpellier, France 


\section{LUDOVIC TEMPLE}

Innovation, Université de Montpellier, CIRAD, INRAE, Montpellier SupAgro, Montpellier, France CIRAD, UMR Innovation, F-34398 Montpellier, France 